\title{
Waiting time effect for motion by positive second derivatives and applications
}

\author{
Qing Liu
}

\begin{abstract}
We discuss the waiting time effect for the evolution of a planar graph governed by its positive part of second derivative. For any smooth periodic function which contains finitely many convex pieces in one period, we show that the waiting time is continuous by using comparison arguments. Moreover, we show that the convex parts keep expanding in size in a strict manner, which answers an open question posed by Kohn and Serfaty (Commun Pure Appl Math 59:344-407, 2006) in this special case. The results on waiting time effect are also applied to the stationary problem of mean curvature type on an unbounded nonconvex domain for our study of its game-theoretic interpretation.
\end{abstract}

Mathematics Subject Classification (1991). 49L25, 35J93, 35K93, 49N90.

Keywords. Viscosity solutions, Deterministic games, Curvature flow equations.

\section{Introduction}

In this paper, we study the motion of a function driven by the positive part of its second derivative. The simplest example is as follows. Suppose $u(x, t)$ is a function defined on $\mathbb{R} \times(0, \infty)$ and satisfies

$$
\begin{cases}u_{t}-\left(u_{x x}\right)_{+}=0 & \text { in } \mathbb{R} \times(0, \infty), \\ u(x, 0)=u_{0}(x) & \text { in } \mathbb{R}\end{cases}
$$

where $a_{+}$denotes $\max \{a, 0\}$ for any $a \in \mathbb{R}$. It is a special case of second-order Hamilton-Jacobi-Bellman equations related to optimization problem of the controlled Brownian motion. In spite of the seemingly simple structure, (1.1) is actually a fully nonlinear, degenerate elliptic equation of non-divergence form. But the standard viscosity solution theory still applies. We get the existence and uniqueness of solutions without much difficulty; see [6,11] and [12]. 
We focus our attention to the geometric properties of the viscosity solution. Notice that the equation will be turned into the usual heat equation provided that the solution $u(x, t)$ is known to be convex in the variable $x$; in particular, when the initial data $u_{0}$ is convex, then the convexity is preserved during the whole evolution, as proved in [13] and [18] etc. On the other hand, if $u_{0}$ is concave, then it is clear that $u_{0}$ itself is the unique stationary solution of (1.1).

A general problem remains incomplete about the situation when the convex and concave pieces both exist initially. In this case, a reasonable result should be that the convex part of the curve moves immediately as in the former case while the nonconvex part stays at the initial position for a while before starting to evolve, which is the so called waiting time effect. One may actually define the waiting time for any point on the graph of $u_{0}$, which we denote by $T_{0}$. Then our prediction above amounts to saying that $T_{0}(z)=0$ when $z$ is on the convex part of $u_{0}$ and $T_{0}(z)>0$ when $z$ is on the concave part. In this paper, we intend to give rigorous and precise descriptions of the waiting time, especially its continuity in space. The key turns out to be the investigation of the motion of inflection points of $u_{0}$.

\subsection{Motion by the positive part of second derivative}

In general the distribution and structure of the inflection points of $u_{0}$ could be very complicated but in order to simplify our introduction and grasp the essence of our problem, we pick a quite special but typical nonconvex initial graph. We first assume that $u_{0}$ is periodic and of $C^{2}$ class. Let $I=(a, b)$ be one period of $u_{0}$ such that $u_{0}(a)=u_{0}(b)=\max _{\mathbb{R}} u_{0}$. For the moment, we add a condition of "unique convex piece", saying that $u_{0}$ is divided by only two inflection points at $x=\alpha$ and $x=\beta(\alpha<\beta)$ so that $u_{0}$ is (strictly) convex on $(\alpha, \beta)$ and (strictly) concave on $I \backslash[\alpha, \beta]$. See Fig. 1. Under the assumptions above, we consider the following general one-dimensional problem:

$$
\begin{cases}u_{t}-h\left(u_{x}\right)\left(u_{x x}\right)_{+}=0 & \text { in } \mathbb{R} \times(0, \infty), \\ u(x, 0)=u_{0}(x) & \text { in } \mathbb{R},\end{cases}
$$

where $h$ is a given function satisfying

(A1) $h \in C(\mathbb{R})$ and $\min _{|p| \leq R} h(p)>0$ for any $R>0$.

This assumption is used to exclude the degeneracy caused by $h$. It is clear that (1.1) is its special case.

We now state the simplest version of our main results on the waiting time.

Theorem 1.1. Assume (A1). Let $u$ be the solution of (1.2) with periodic initial condition $u_{0}$ of class $C^{2}$. Let $\alpha_{t}$ and $\beta_{t}$ stand for the $x$-coordinates of the only two inflection points of $u(x, t)$ in a period $[a, b]$ at each time $t>0$ with $\alpha_{0}=\alpha$ and $\beta_{0}=\beta$. Then the following statements hold:

(1) $\alpha_{t}$ and $\beta_{t}$ are continuous in $t$;

(2) $\alpha_{t}$ is strictly decreasing and $\beta_{t}$ is strictly increasing in $t$;

(3) $\alpha_{t} \rightarrow a$ and $\beta_{t} \rightarrow b$ as $t \rightarrow \infty$. 


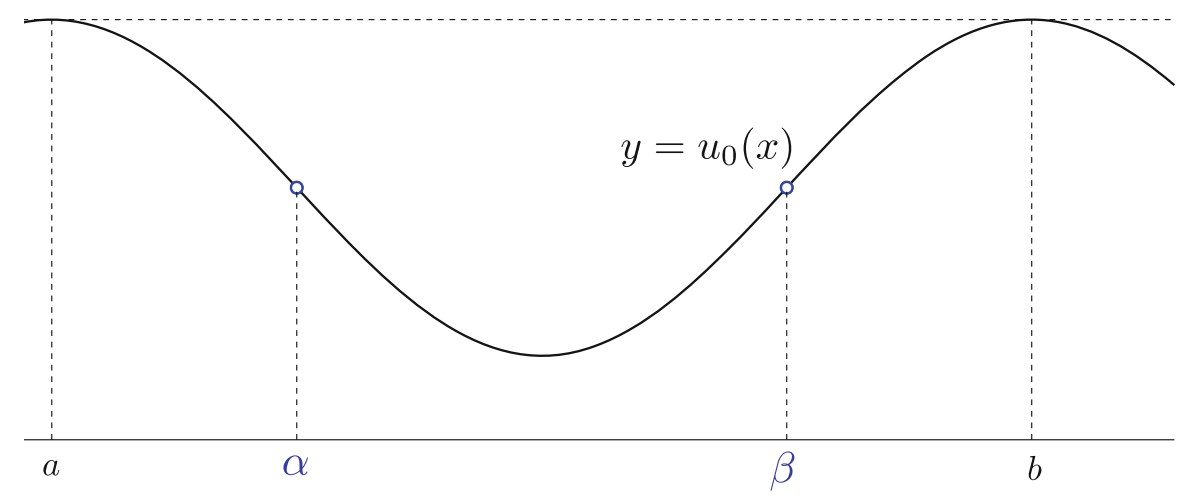

Figure 1. The initial graph $y=u_{0}(x)$

Since $\left(\alpha_{t}, \beta_{t}\right)$ consists of the locations of all convex points of $u(x, t)$ at $t$, the above consequence amounts to saying that the convex parts of $u$ keep expanding in a strict manner. As a result, we easily obtain the continuity of the waiting time $T_{0}$ with respect to $x$. In Sect. 3 , we give a more general version of Theorem 1.1. The key ingredient is the investigation of the motion of all inflection points. Note that in this general setting the inflection points may collide each other during the evolution.

\subsection{Motivation: the game interpretation of mean curvature flow}

Let us introduce the motivation of the above analysis about the waiting time. We first remark that the equation

$$
\begin{cases}u_{t}-\frac{\left(u_{x x}\right)_{+}}{1+u_{x}^{2}}=0 & \text { in } \mathbb{R} \times(0, \infty), \\ u(x, 0)=u_{0}(x) & \text { in } \mathbb{R}\end{cases}
$$

is another special case of (1.2). This equation is the graph formulation of the socalled motion by positive curvature, whose corresponding level-set formulation is as follows:

$$
\begin{cases}U_{t}-|\nabla U| \operatorname{div}\left(\frac{\nabla U}{|\nabla U|}\right)_{+}=0 & \text { in } \mathbb{R}^{2} \times(0, \infty), \\ U(x, 0)=U_{0}(x) & \text { in } \mathbb{R}^{2},\end{cases}
$$

where $U_{0}$ is a defining function of the initial curve. This equation is a little different from the normal mean curvature flow equation, which is independently studied in [4] and [8]. We refer to [12] for details on the well-posedness of (E1) in the framework of viscosity solutions. It also has applications in image processing [21]. We remark that waiting time effect was studied for other geometric motions. For example, one may refer [5] for this phenomenon for Gauss curvature flows. Our equation is clearly different from theirs. 
Our waiting time results partially answered an open problem proposed by Kohn and Serfaty [16] about (E1). They asked whether or not for any nonconvex curve in the plane there exists a free boundary separating the (moving) convex part and the (stationary) concave part. They also asked whether it is true that the concave part decreases monotonically in size and any part of the curve never stops once it starts to move. It is known that in general the answers are negative if no regularity conditions more than Lipschitz continuity are assumed; see the example given by G. Barles and F. Da Lio in [16, Appendix C.3]. We give affirmative answers to all of these questions for any smooth periodic graph with finitely many concave pieces.

Another closely related motivation is to study the deterministic game interpretation for the stationary counterpart of (E1) also proposed by Kohn and Serfaty [16]. For a bounded domain $\Omega \subset \mathbb{R}^{2}$, they introduced a family of exit time games with a parameter $\epsilon>0$, whose value functions $T^{\epsilon}(z)$ converge, under several conditions on $\Omega$, to the solution $T$ of the stationary problem of mean curvature type:

$$
\begin{cases}-|\nabla T| \operatorname{div}\left(\frac{\nabla T}{|\nabla T|}\right)-1=0 & \text { in } \Omega, \\ T=0 & \text { on } \partial \Omega,\end{cases}
$$

which is sometimes called normalized 1-Laplace equation. This approximation gives an interesting representation of the solutions to the equation. See also [17] for the deterministic game approach to general elliptic and parabolic equations and [22-25] for a stochastic tug-of-war game approach to the $p$-Laplace equation with $p>1$. Related extensions of this new method to the Heisenberg group are recently addressed in $[9,10]$.

More precisely, it is proved in [16] that the relaxed semi-limits $\bar{T}$ and $\underline{T}$ are respectively a subsolution and a supersolution of (E2) and it suffices to use the comparison principle to conclude the convergence $T^{\epsilon} \rightarrow T$ as $\epsilon \rightarrow 0$.

The usual comparison theorem to guarantee the uniqueness of continuous solutions of this Dirichlet problem is known only when $\Omega$ is convex [8] and the desired convergence above follows easily in this case. It is however less complete when the convexity of $\Omega$ is dropped. Without any assumptions on the convexity or regularity of $\Omega$, the solutions can easily become discontinuous, as is shown again in the example of G. Barles and F. Da Lio in [16, Appendix C.3]. The Dirichlet boundary condition may not be realized in the strict sense and therefore the usual comparison principle does not hold.

The best one can expect in the nonconvex case seems to be a unique result for possibly discontinuous solutions by showing the so-called weak comparison principle for solutions with boundary condition interpreted in the viscosity sense; namely, if $U$ and $V$ are respectively a subsolution and a supersolution with the boundary condition (1.7) in the viscosity sense, then $U_{*} \leq V_{*}$ and $U^{*} \leq V^{*}$ in $\bar{\Omega}$, where $W_{*}$ and $W^{*}$ are respectively the lower and upper semicontinuous envelopes of any bounded function $W$. Applying this weaker comparison result, one may obtain the game approximation for the possibly discontinuous solution and the convergence is certainly in a weaker sense. 


\subsection{Application of results for waiting time}

The above weak approach does work but it requires $\Omega$ to be star-shaped [16]. Without the star-shapedness, even the weak comparison principle is not necessarily true. An example involving $\Omega$ of figure-eight type is provided in [19], where a necessary condition, related to the fattening phenomenon, for the weak comparison principle is given as well.

In this work, we will show that $T^{\epsilon}$ may actually converge to a continuous solution of (1.6) in a nonconvex (unbounded) domain. We prove the convergence for the case when $\partial \Omega$ is represented by the graph of a function $u_{0}$ satisfying the periodicity and having finitely many smooth concave parts in each period, as was described previously. In this special case, we prove that the solution will become continuous. This improvement is obtained mainly due to the improved regularity of the boundary $\partial \Omega$.

Our method is to pose stronger boundary conditions which the limit of $T^{\epsilon}$ satisfies. The main difference from [16] lies in the following two aspects.

1. We stress that under our assumptions on $\partial \Omega$, the domain $\Omega$ is unbounded. The problem on solvability of (E2) in an unbounded domain seems to be new to our best knowledge. We overcome this difficulty by linking the value functions $T^{\epsilon}$ with the game values approximating the solution of (E1). We are able to determine an effective domain. In fact, we show that $\Gamma:=\left\{(x, y) \in \mathbb{R}^{2}: y=\max u_{0}\right\}$ divides $\Omega$ into a half plane, in which $T^{\epsilon}=\infty$, and infinitely many congruent bounded regions, where $T^{\epsilon}$ is locally bounded. Let $\Omega_{e}$ be one of the region. We also show that $T^{\epsilon}(z) \rightarrow \infty$ when $z \in \Omega_{e}$ satisfies $\operatorname{dist}(z, \Gamma) \rightarrow 0$. This means that a singular Dirichlet boundary condition on $\Gamma$ appears in the limit PDE problem. Singular boundary problems are studied only for second-order semilinear equations by Lasry and Lions [20] and for first-order Hamilton-Jacobi equations in [1,3,7] with applications to large time asymptotics in $[14,15]$. Our singular boundary problem is different from all these works, since our equation is quasilinear and degenerate elliptic.

2. As the boundary of $\Omega_{e}$ is composed of $\Gamma$ and $\partial \Omega$. We need to give another boundary condition on $\partial \Omega$. It turns out that the limiting boundary value of $T^{\epsilon}$ as $\epsilon \rightarrow 0$ is nothing else but the waiting time $T_{0}$ of (1.3). Here we apply our continuity results about the waiting time. The game interpretation of (E1) with $U_{0}$ being a defining function of $\partial \Omega$ is used again to connect (1.3) and (E2).

With the preparation above, we can characterize the limit of $T^{\epsilon}$ as the unique continuous solution $T$ of the following problem with mixed boundary conditions:

$$
\begin{cases}-|\nabla T| \operatorname{div}\left(\frac{\nabla T}{|\nabla T|}\right)-1=0 & \text { in } \Omega_{e}, \\ T(z)=T_{0}(z) & \text { for all } z \in \partial \Omega, \\ T(z) \rightarrow \infty & \text { as } \operatorname{dist}(z, \Gamma) \rightarrow 0,\end{cases}
$$


where $T_{0}$ is the waiting time we obtained previously. Note that (1.8) is now interpreted in the strict sense and therefore the usual comparison theorem follows easily. We prove that $T^{\epsilon} \rightarrow T$ uniformly in any compact subset of $\Omega_{e}$. By using the weak approach as in [16], we may show that the solution $T$ of (E3) is also a solution of

$$
\begin{cases}-|\nabla T| \operatorname{div}\left(\frac{\nabla T}{|\nabla T|}\right)-1=0 & \text { in } \Omega_{e} \\ T(z)=0 & \text { for all } z \in \partial \Omega \\ T(z) \rightarrow \infty & \text { as } \operatorname{dist}(z, \Gamma) \rightarrow 0\end{cases}
$$

where (1.10) is fulfilled in the viscosity sense. This indicates that if one can follow [16] to get a unique weak solution of (E4), then it must be continuous and satisfy (E3). We are not able to show the uniqueness of weak solutions but a comparison principle is given to show that the game-related continuous solution $T$ is the biggest among all of the weak solutions in the effective domain. Indeed, our comparison principle, whose proof is similar to the argument used for state constraint problems [26], states that any upper semicontinuous subsolution is not above any continuous supersolution. The uniqueness of weak solutions will be completed under current assumptions if we can show a symmetric comparison result, which says that any continuous subsolution is not larger than any lower semicontinuous supersolution. We give a very simple example, revealing that this is impossible without making any extra assumptions on the accessibility of the supersolution.

We finally remark that it is possible to extend our arguments to a bounded domain with analogous regularity by applying the analysis of waiting time locally.

This paper is organized in the following way. In Sect. 2 we briefly introduce some basic properties of the Eq. (1.2). In Sect. 3 we study in detail the waiting time effect and show its continuity. We prove a general version of Theorem 1.1. The application to the stationary level-set equation of mean curvature type is presented in Sect. 4. We establish the associated games and show the convergence of game values to the unique continuous solution of the stationary problem. Some discussions including a comparison principle are also presented for the maximality of the continuous solution among all weak solutions.

Notation. In this article, we use the following notations.

For any $z \in \mathbb{R}^{n}$ and $r>0$, we use $B_{r}(z)$ to denote the open ball in $\mathbb{R}^{n}$ centered at $z$ with radius $r$.

For any $z_{1}, z_{2} \in \mathbb{R}^{2}$, we denote by $\overline{z_{1} z_{2}}$ the line segment between $z_{1}$ and $z_{2}$, i.e.,

$$
\overline{z_{1} z_{2}}:=\left\{k z_{1}+(1-k) z_{2}: k \in[0,1]\right\} .
$$

For any function $u: \mathbb{R}^{n+1} \rightarrow \mathbb{R}$ of class $C^{2}$, we use $u_{x_{i}}, u_{t}$ and $u_{x_{i} x_{j}}$ to denote the partial derivatives of $u$, i.e., $u_{x_{i}}:=\partial u / \partial x_{i}, u_{t}:=\partial u / \partial t$ and $u_{x_{i} x_{j}}=\partial^{2} u / \partial x_{i} x_{j}$ for all $i, j=1,2, \ldots, n$. We denote the gradient of $u$ in 
space by $\nabla u:=\left(\partial u / \partial x_{1}, \partial u / \partial x_{2}, \ldots, \partial u / \partial x_{n}\right)$. We also write $u^{\prime}$ to represent the derivative $d u / d x$ provided that $u$ is a function of one variable $x$.

\section{Motion by positive second derivatives}

Let us begin with a brief review of some basic results about the Eq. (1.2), which gives a general description of the motion of a graph by its positive second derivative in one dimension. With the condition (A1), we find that (1.2) is a fully nonlinear degenerate parabolic equation. For any classical solution $u \in C^{2}(\mathbb{R} \times(0, \infty)), u$ satisfies

$$
u_{t}-h\left(u_{x}\right) u_{x x}=0 \quad \text { when } u_{x x} \geq 0
$$

but

$$
u_{t}=0 \quad \text { when } u_{x x}<0,
$$

which reveals that (1.2) is actually a combination of a parabolic second-order equation and a (trivial) first order equation and the type change occurs at the inflection points of $u$. It is also clear that (1.3) is a special case of (1.2).

In spite of the nonlinearity, we can still get a unique solution in the framework of viscosity solution theory.

Theorem 2.1. Assume (A1). Let $u_{0}$ be a Lipschitz continuous function on $\mathbb{R}$. Then there exists a unique viscosity solution u of (1.2). Moreover, $u$ is Lipschitz continuous in space.

We refer to [6] and [12] for the definition of viscosity solutions and the proof for Theorem 2.1.

The solution of (1.2) obviously enjoys monotonicity in time.

Lemma 2.2. Assume (A1). Let $u_{0}$ be a Lipschitz continuous function on $\mathbb{R}$. Let $u$ be the solution of (1.2). Then $u(x, t) \geq u(x, s)$ for any $x \in \mathbb{R}$ and $t \geq s \geq 0$.

Since $u$ satisfies

$$
u_{t} \geq 0 \quad \text { in } \mathbb{R} \times(0, \infty)
$$

in the viscosity sense, we can easily show the monotonicity by following $[2$, Lemma 5.15] or [15, Lemma 4.4].

Remark 2.1. It is easily seen that Lemma 2.2 can be extended to CauchyDirichlet problems. The proof is analogous and therefore omitted here as well.

We assume the following periodicity in space for simplicity.

(A2) $u_{0}$ is a periodic Lipschitz continuous function on $\mathbb{R}$. Let $I:=(a, b)$ denote one of its open periods satisfying

$$
u_{0}(a)=u_{0}(b)=\max _{x \in \mathbb{R}} u_{0}(x)
$$

and

$$
u_{0}<M \quad \text { in } I
$$


We present all our analysis in the interval $I$. Denote by $\Omega_{0}$ the epigraph of $u_{0}$ in $I$, i.e.,

$$
\Omega_{0}:=\left\{(x, y) \in \mathbb{R}^{2}: y>u_{0}(x), x \in I\right\} .
$$

Let $M:=\max _{x \in \mathbb{R}} u_{0}(x)$ and $m=\min _{x \in \mathbb{R}} u_{0}(x)$. Note that the constant $M$ is a supersolution, which, by comparison principle, implies that the solution $u$ satisfies $u(a, t)=u(b, t)=M$ for all $t \geq 0$.

The large-time behavior of (1.3) is quite simple for this specific initial value.

Proposition 2.3. (Large-time behavior) Assume (A1) and (A2). Let $u$ be the solution of (1.2). Then $u(x, t) \rightarrow M=\max _{x \in \mathbb{R}} u_{0}(x)$ uniformly for all $x \in \mathbb{R}$ as $t \rightarrow \infty$.

Proof. One may first take the relaxed limits of $u$ as $t \rightarrow \infty$ :

$$
\bar{u}(x)=\limsup _{t \rightarrow \infty} * u(x, t) \quad \text { and } \quad \underline{u}(x)=\liminf _{t \rightarrow \infty} u(x, t) .
$$

Since the constant $M$ is a supersolution and by Lemma $2.2 u$ is nondecreasing in $t$, we have $u_{0}(x) \leq u(x, t) \leq M$, which implies that $u_{0} \leq \underline{u} \leq \bar{u} \leq M$ in $\mathbb{R}$. In particular, we have $\bar{u}(a)=\underline{u}(a)=\bar{u}(b)=\underline{u}(b)=M$. Hence by the standard stability theory for viscosity solutions, we may prove $\underline{u}$ in $I$ is a supersolution of

$$
\left\{\begin{array}{l}
-h\left(u_{x}\right)\left(u_{x x}\right)_{+}=0 \quad \text { in } I=(a, b) \\
u(a)=u(b)=M .
\end{array}\right.
$$

We assert that $\underline{u}=M$ in $I$. Indeed, if there exists $x_{0} \in I$ such that $\underline{u}\left(x_{0}\right)<M$, then we may find a smooth (quadratic) function $\phi$ in $[a, b]$ satisfying $\phi(a)=$ $\phi(b)=M, \underline{u}\left(x_{0}\right)<\phi\left(x_{0}\right)<M$ and $\phi_{x x}>0$ in $I$. It is obvious that there exists $\bar{x} \in I$ such that

$$
\min _{x \in[a, b]}(u-\phi)(x)=u(\bar{x})-\phi(\bar{x})<0 .
$$

By the definition of viscosity supersolutions, we get

$$
-h\left(\phi_{x}(\bar{x})\right)\left(\phi_{x x}(\bar{x})\right)_{+} \geq 0,
$$

which yields that $\phi_{x x}(\bar{x}) \leq 0$. This contradicts the fact that $\phi_{x x}>0$ in $I$.

It is now clear that $\bar{u}=\underline{u}=M$, which implies the uniform convergence of $u(x, t)$ to the constant solution of $(2.2)$ as $t \rightarrow \infty$.

\section{Waiting time effect}

A very special property of the motion by positive second derivatives (1.2) is its waiting time before moving.

We divide the period $I$ into a convex part, a concave part and the remaining part. In order to further simplify our exposition and show the essence of our argument, let us make the following assumptions.

(A3) Let $I_{0}=\cup_{i=1}^{m}\left\{\alpha_{0}^{i}, \beta_{0}^{i}\right\}$, where $m \in \mathbb{Z}$ and $\alpha_{0}^{i}, \beta_{0}^{i} \in I$ such that

(a) $\alpha_{0}^{i}<\beta_{0}^{i}<\alpha_{0}^{i+1}<\beta_{0}^{i+1}$ for all $i=1,2, \ldots, m-1$; 
(b) $u_{0}$ is convex in $I_{+}=\cup_{i=1}^{m}\left(\alpha_{0}^{i}, \beta_{0}^{i}\right)$;

(c) $u_{0}$ is of class $C^{1}$ in $I_{-}:=I \backslash\left(I_{+} \cup I_{0}\right)$ with $u_{0}^{\prime}$ strictly decreasing in each interval of $I_{-}$;

(d) For any $\alpha_{0}^{i}, \beta_{0}^{i} \in I_{0}$,

$$
\begin{aligned}
k_{0}^{\alpha, i} & :=\lim _{x \rightarrow \alpha_{0}^{i}-} \frac{u_{0}(x)-u_{0}\left(\alpha_{0}^{i}\right)}{x-\alpha_{0}^{i}} \leq \lim _{x \rightarrow \alpha_{0}^{i}+} \frac{u_{0}(x)-u_{0}\left(\alpha_{0}^{i}\right)}{x-\alpha_{0}^{i}} \text { and } \\
k_{0}^{\beta, i}: & :=\lim _{x \rightarrow \beta_{0}^{i}+} \frac{u_{0}(x)-u_{0}\left(\beta_{0}^{i}\right)}{x-\beta_{0}^{i}} \geq \lim _{x \rightarrow \beta_{0}^{i}-} \frac{u_{0}(x)-u_{0}\left(\beta_{0}^{i}\right)}{x-\beta_{0}^{i}} ;
\end{aligned}
$$

(e) $k_{0}^{\alpha, i}<k_{0}^{\beta, i}$ for any $i=1,2, \ldots, m$.

For our convenience of notation, we let $\alpha_{0}^{m+1}=b$ and $\beta_{0}^{0}=a$. The assumptions (a)-(c) give a partition of the graph by convexity. Note that we do not explicitly assume any regularity, more than Lipschitz continuity, of the convex part $I_{+}$but we assume some relation between the left and right derivatives at the inflection points in (d), which, together with (a)-(c), implies that $u_{0}$ is semiconvex. The assumption (e) roughly states that each convex piece $\left(\alpha_{0}^{i}, \beta_{0}^{i}\right)$ contains strict convex points.

It is easy to see that a smooth graph with finitely many convex and concave pieces fulfills (A3). Since $\alpha_{0}^{i}$ and $\beta_{0}^{i}$ correspond to the inflection points in the smooth case, we still call them inflection points in our general setting (A3) as well.

\subsection{Existence of waiting time}

We first show the general existence of waiting time effect. Similar results for (E1) are shown in [16, Theorem 1.7] by a game approach.

Lemma 3.1. (Existence of waiting time) Assume (A1)-(A3). Let $u$ be the solution of (1.2). For any $x \in I_{-}$, there exists $\tau_{x}>0$ depending on $x$ such that

$$
u(x, t)=u_{0}(x) \quad \text { for all } t \in\left[0, \tau_{x}\right] .
$$

Proof. Fix an arbitrary $x_{0} \in I_{-}$and take $w(x, s)=B x+C$ with $B, C \in \mathbb{R}$ properly chosen so that there exist $x_{1}, x_{2} \in I_{-}$satisfying $x_{1}<x_{0}<x_{2}$, $w\left(x_{0}, 0\right)=u_{0}\left(x_{0}\right)$ and

$$
w(x, 0)>u_{0}(x) \text { for all } x \in\left[x_{1}, x_{0}\right) \cup\left(x_{0}, x_{2}\right] .
$$

By continuity of the solution $u(x, t)$, there exists $\tau>0$ such that $u\left(x_{1}, s\right)<$ $w\left(x_{1}, 0\right)$ and $u\left(x_{2}, s\right)<w\left(x_{2}, 0\right)$ for any $s \in[0, \tau]$. Hence, $w$ is a supersolution of

$$
\left\{\begin{array}{l}
v_{s}-h\left(v_{x}\right)\left(v_{x x}\right)_{+}=0 \quad \text { in }\left(x_{1}, x_{2}\right) \times(0, \tau) \\
v\left(x_{1}, s\right)=u\left(x_{1}, s\right) \text { and } v\left(x_{2}, s\right)=u\left(x_{2}, s\right) \text { for all } s \in[0, \tau] \\
v(x, 0)=u_{0}(x) \text { for all } x \in\left[x_{1}, x_{2}\right]
\end{array}\right.
$$

The comparison theorem yields that $u\left(x_{0}, s\right) \leq w\left(x_{0}, s\right) \equiv u_{0}\left(x_{0}\right)$ and therefore $u\left(x_{0}, s\right)=u_{0}\left(x_{0}\right)$ for any $s \in[0, \tau]$ by the monotonicity of $u$ in time derived in Lemma 2.2. 
For any $z_{0}=\left(x_{0}, u_{0}\left(x_{0}\right)\right) \in \mathbb{R}^{2}$, define

$$
T_{0}\left(z_{0}\right):=\sup \left\{\tau: u\left(x_{0}, t\right)=u_{0}\left(x_{0}\right) \text { for any } t \leq \tau\right\}
$$

We call $T_{0}\left(z_{0}\right)$ the waiting time of the curve $y=u_{0}(x)$ at $z_{0}$. Note that under the assumptions (A1)-(A3), for any $x \in I$ such that $u_{0}(x)<M=$ $\lim _{t \rightarrow \infty} u(x, t)$, we have $T_{0}(x)<\infty$ by Proposition 2.3 .

\subsection{Short time convexity of moving pieces}

In what follows, we investigate the behavior of inflection points when time $t$ is small. Define for any $i=0,1,2, \ldots, m$

$$
\begin{aligned}
& \alpha_{t}^{i+1}:=\sup \left\{x: u(x, t)=u_{0}(x), \quad \beta_{0}^{i} \leq x \leq \alpha_{0}^{i+1}\right\}, \\
& \beta_{t}^{i}:=\inf \left\{x: u(x, t)=u_{0}(x), \quad \beta_{0}^{i} \leq x \leq \alpha_{0}^{i+1}\right\} .
\end{aligned}
$$

Lemma 3.1 asserts that the definitions above are valid and $\beta_{t}^{i}<\alpha_{t}^{i+1}$ when $t>0$ is sufficiently small. For each $i=0,1, \ldots, m$, let

$$
\tau_{i}:=\sup \left\{t \in[0, \infty): \beta_{t}^{i}<\alpha_{t}^{i+1}\right\} .
$$

It is therefore clear that $\tau_{i}>0$ for all $i=0,1, \ldots, m$. Denote

$$
\tau^{*}:=\min _{i=0,1, \ldots, m} \tau_{i}=\sup \left\{t \in[0, \infty): \beta_{t}^{i}<\alpha_{t}^{i+1} \text { for all } i=1,2, \ldots, m\right\},
$$

which gives a more precise bound in $t$ for which the definitions in (3.3) are valid. Moreover, Lemma 2.2 implies the monotonicity of $\alpha_{t}^{i+1}$ and $\beta_{t}^{i}$; that is,

$$
\alpha_{t}^{i+1} \leq \alpha_{s}^{i+1} \text { and } \quad \beta_{t}^{i} \geq \beta_{s}^{i} \quad \text { for all } 0 \leq s \leq t<\tau^{*} .
$$

We may also define $\alpha_{\tau^{*}}^{i+1}$ and $\beta_{\tau^{*}}^{i}$ by letting $t=\tau^{*}$ in (3.3). In fact,

$$
\alpha_{\tau^{*}}^{i+1}=\lim _{t \rightarrow \tau^{*}} \alpha_{t}^{i+1} \quad \text { and } \quad \beta_{\tau^{*}}^{i}=\lim _{t \rightarrow \tau^{*}} \beta_{t}^{i}
$$

due to the continuity of the solution $u$.

Remark 3.1. We remark that $\tau^{*}$ can be $\infty$. When $\tau^{*}=\infty$, we must have the following situation: for any $i=0,1, \ldots, m$, there exists $x_{i} \in\left[\beta_{0}^{i}, \alpha_{0}^{i+1}\right]$ such that $u_{0}\left(x_{i}\right)=M=\max _{x \in I} u_{0}(x)$. Indeed, suppose by contradiction that there exists some $i$ such that $u_{0}(x)<M-\epsilon$ with $\epsilon>0$ for all $x \in\left[\beta_{0}^{i}, \alpha_{0}^{i+1}\right]$. Then by (3.3) and the fact that $\tau^{*}=\infty$, we obtain that $\emptyset \neq\left(\beta_{t}^{i}, \alpha_{t}^{i+1}\right) \subset\left[\beta_{0}^{i}, \alpha_{0}^{i}\right]$ for all $t>0$, which yields the existence of a certain $x(t) \in\left(\beta_{t}^{i}, \alpha_{t}^{i+1}\right)$ such that $u(x(t), t)=u_{0}(x(t))<M+\epsilon$. By taking a subsequence, we may let $x(t) \rightarrow x_{\infty} \in I$ as $t \rightarrow \infty$. By Proposition 2.3, we end up with $u_{0}\left(x_{\infty}\right)=M$, which is a contradiction.

We next study the motion of all inflection points when $t<\tau^{*}$.

Remark 3.2. Under the assumptions (A1)-(A3), we may easily obtain for any $t<\tau^{*}$ and any $i=0,1, \ldots, m$

$$
u(x, s)=u_{0}(x) \quad \text { for all } x \in\left(\beta_{t}^{i}, \alpha_{t}^{i+1}\right) \text { and } s \in[0, t) .
$$

Indeed, from the definition of $\alpha_{t}^{i+1}$ and $\beta_{t}^{i}$ and the continuity of $u$, we have $u\left(\alpha_{t}^{i+1}, t\right)=u_{0}\left(\alpha_{t}^{i+1}\right)$ and $u\left(\beta_{t}^{i}, t\right)=u_{0}\left(\beta_{t}^{i}\right)$, which by Lemma 2.2 implies that $u$ stays stationary at $\alpha_{t}^{i+1}$ and $\beta_{t}^{i}$ before time $t$; in other words, $u\left(\alpha_{t}^{i+1}, s\right)=$ 
$u_{0}\left(\alpha_{t}^{i+1}\right)$ and $u\left(\beta_{t}^{i}, s\right)=u_{0}\left(\beta_{t}^{i}\right)$ for all $0 \leq s \leq t$. Noting that $u(a, s)=u(b, s)=$ $u_{0}(a)=u_{0}(b)=M$ for all $s \geq 0$, we find that $u(x, s)$ is actually a solution of

$$
\begin{cases}u_{s}-h\left(u_{x}\right)\left(u_{x x}\right)_{+}=0 & \text { in }\left(\beta_{t}^{i}, \alpha_{t}^{i+1}\right) \times(0, t), \\ u\left(\beta_{t}^{i}, s\right)=u_{0}\left(\beta_{t}^{i}\right) \text { and } u\left(\alpha_{t}^{i+1}, s\right)=u_{0}\left(\alpha_{t}^{i+1}\right) & \text { for all } s \in[0, t], \\ u(x, 0)=u_{0}(x) & \text { for all } x \in\left[\beta_{t}^{i}, \alpha_{t}^{i+1}\right] .\end{cases}
$$

We are thus led to (3.5) due to the fact that $u_{0}$ is the unique solution of the above problem.

Theorem 3.2. (Continuity in time of inflection points) Assume (A1)-(A3). Let $u$ be the solution of (1.2). Let $\alpha_{t}^{i}, \beta_{t}^{i}$ be defined as in (3.3) for all $i=1,2, \ldots, m$ and $t \in\left[0, \tau^{*}\right)$, where $\tau^{*}$ is given in (3.4). Then $\alpha_{t}, \beta_{t}:\left[0, \tau^{*}\right) \rightarrow I$ are both continuous functions.

Proof. Let us only show the continuity for $\beta_{t}^{i}$ with any fixed $i=1,2, \ldots, m$. We fix $t_{0} \in\left[0, \tau^{*}\right)$. Our goal is to show $\lim _{t \rightarrow t_{0}} \beta_{t}^{i}=\beta_{t_{0}}^{i}$. Notice that $\beta_{t_{0}}^{i}<\alpha_{t_{0}}^{i+1}$, since $t<\tau^{*}$.

1. We first show the right continuity, i.e.,

$$
\lim _{t \rightarrow t_{0}+} \beta_{t}^{i}=\beta_{t_{0}}^{i}
$$

Indeed, by the monotonicity of $\beta_{t}^{i}$ in $t$, we have $\liminf _{t \rightarrow t_{0}+} \beta_{t} \geq \beta_{t_{0}}$. On the other hand, by Remark 3.2, $u\left(x, t_{0}\right)=u_{0}(x)$ for all $x \in\left(\beta_{t_{0}}^{i}, \alpha_{t_{0}}^{i+1}\right)$. Set $\tilde{u}(x, t)=u\left(x, t+t_{0}\right)$. Then $\tilde{u}$ is the solution of (1.2) with initial data $u\left(x, t_{0}\right)$. Using Lemma 3.1 for $\tilde{u}$ and any $x \in\left(\beta_{t_{0}}^{i}, \alpha_{t_{0}}^{i+1}\right)$, we may take $\tau=t-t_{0}>0$ small such that $\tilde{u}(x, s)=u\left(x, t_{0}\right)$ for all $s \in[0, \tau]$, which yields that $u(x, t)=u\left(x, \tau+t_{0}\right)=u_{0}(x)$. We therefore get $\beta_{t}^{i}<x$ as long as $t>t_{0}$ is sufficiently close to $t_{0}$. If follows that $\limsup _{t \rightarrow t_{0}+} \beta_{t}^{i} \leq \beta_{t_{0}}^{i}$.

2. We next consider the left continuity. Thanks to the monotonicity of $\alpha_{t}^{i+1}$ and $\beta_{t}^{i}$ in $t$, we may assume by contradiction that there exists an increasing sequence $t_{n} \rightarrow t_{0}-$ as $n \rightarrow \infty$ such that

$$
\lim _{n \rightarrow \infty} \beta_{t_{n}}^{i}<\beta_{t_{0}}^{i}-\delta_{0} \quad \text { for some } \delta_{0}>0
$$

and

$$
\lim _{n \rightarrow \infty} \alpha_{t_{n}}^{i+1} \geq \alpha_{t_{0}}^{i+1}
$$

It follows from Remark 3.2 that $u\left(x, t_{n}\right)=u_{0}(x)$ for all $x \in\left[\beta_{t_{n}}^{i}, \alpha_{t_{n}}^{i+1}\right)$, which, by continuity of $u$, implies that $u\left(x, t_{n}\right)=u_{0}(x)$ and therefore $u\left(x, t_{0}\right)=u_{0}(x)$ for all $x \in\left[\beta_{t_{0}}^{i}-\delta_{0}, \alpha_{t_{0}}^{i+1}\right)$. This contradicts the definition of $\beta_{t_{0}}^{i+1}$.

Remark 3.3. The second half of our proof above can be used to show that there exists $i=0,1,2, \ldots, m$ such that $\alpha_{\tau^{*}}^{i+1}=\beta_{\tau^{*}}^{i}$. Indeed, there is $i=0,1,2, \ldots, m$ such that $\tau^{*}=\tau_{i}$. Fix such $i$. By definition, we easily get $\beta_{\tau^{*}}^{i} \leq \alpha_{\tau^{*}}^{i+1}$. Both sides are actually equal, for otherwise we may utilize the existence result of 
waiting time, Lemma 3.1, for the points between them and the initial time $t=\tau^{*}$ to derive a contradiction that $\tau_{i}>\tau^{*}$.

Lemma 3.3. (Support functions at inflection points) Assume (A1)-(A3). Let $u$ be the solution of (1.2). For any $i=1, \ldots, m$ and $t \in\left[0, \tau^{*}\right)$, let $y=L_{t}^{\alpha, i}(x)$ and $y=L_{t}^{\beta, i-1}(x)$ be the equations of tangent lines to $u_{0}$ respectively at $\alpha_{t}^{i}$ and $\beta_{t}^{i-1}$. Then

$$
u(x, t) \geq \max \left\{L_{t}^{\alpha, i}(x), L_{t}^{\beta, i}(x)\right\} \quad \text { for all } x \in\left[\alpha_{t}^{i}, \beta_{t}^{i}\right] .
$$

Proof. The statement obviously holds when $t=0$ due to (A3). We only show $u(\cdot, t) \geq L_{t}^{\beta, i}(\cdot)$ in $\left[\alpha_{t}^{i}, \beta_{t}^{i}\right]$ for $t>0$. The conclusion follows by using a symmetric argument to prove $u(\cdot, t) \geq L_{t}^{\alpha, i}(\cdot)$ in $\left[\alpha_{t}^{i}, \beta_{t}^{i}\right]$.

Suppose by contradiction that there exists $t>0$ such that there is $x_{1} \in$ $\left[\alpha_{t}^{i}, \beta_{t}^{i}\right]$ satisfying $u\left(x_{1}, t\right)<L_{t}^{\beta, i}\left(x_{1}\right)$, which yields that $u\left(x_{1}, s\right)<L_{t}^{\beta, i}\left(x_{1}\right)$ for any $0 \leq s \leq t$. Then we may take $y_{1} \in\left(u\left(x_{1}, t\right), L_{t}^{\beta, i}\left(x_{1}\right)\right)$.

Take another tangent line $y=L^{*}(x)$ to $u_{0}$ passing through $\left(x_{1}, y_{1}\right)$. We assume that they are tangent at $x=x_{0}$. Then we must have $x_{0}<\beta_{t}^{i}$. We pick $x_{2}$ from the interval $\left(\beta_{t}^{i}, \alpha_{t}^{i+1}\right)$, which is nonempty due to $t<\tau_{i}$, and denote $y_{2}=L^{*}\left(x_{2}\right)$. It is obvious that $u\left(x_{2}, s\right)=u_{0}\left(x_{2}\right)<y_{2}$ for all $s \in[0, t]$.

It is now clear that $y=L^{*}(x)$ is a supersolution of the Cauchy-Dirichlet problem (3.1) with $\tau=t$. Since $u$ is the solution of the same equation, by comparison theorem for $(3.1)$, we have $u(x, s) \leq L^{*}(x)$ for all $x \in\left[x_{1}, x_{2}\right]$ and $s \in[0, t]$, and in particular, $u\left(x_{0}, t\right) \leq L^{*}(x)$. This implies that $u\left(x_{0}, t\right)=$ $u_{0}\left(x_{0}\right)$. On the other hand, by definition of $\beta_{t}^{i}$, we get $u\left(x_{0}, t\right)>u_{0}\left(x_{0}\right)$, which is a contradiction.

Remark 3.4. The conclusion in Lemma 3.3 holds for $t=\tau^{*}$ as well. We only need to send the limit in (3.6) as $t \rightarrow \tau^{*}$ and the continuity of $u$ yields

$$
u(x, t) \geq \max \left\{L_{\tau^{*}}^{\alpha, i}(x), L_{\tau^{*}}^{\beta, i}(x)\right\} \quad \text { for all } x \in\left[\alpha_{\tau^{*}}^{i}, \beta_{\tau^{*}}^{i}\right] .
$$

For any $i=1,2, \ldots, m$ and $t \in\left(0, \tau^{*}\right)$, denote

$$
\Omega_{t}^{i}:=\left\{(x, y) \in \mathbb{R}^{2}: y>u(x, t), \alpha_{t}^{i}<x<\beta_{t}^{i}\right\} .
$$

An immediate consequence of Lemma 3.3 is as follows.

Lemma 3.4. Assume (A1)-(A3). For any $i=1,2, \ldots, m$ and $t \in\left(0, \tau^{*}\right)$, let $\Omega_{0}$ and $\Omega_{t}^{i}$ be defined as in (2.1) and (3.7) respectively. Then for any $z_{1}, z_{2} \in \overline{\Omega_{t}^{i}}$, we have $\overline{z_{1} z_{2}} \subset \overline{\Omega_{0}}$.

Proof. Set

$$
\mathcal{S}:=\left\{(x, y) \in \mathbb{R}^{2}: y \geq L_{t}^{\alpha, i}(x) \vee L_{t}^{\beta, i}(x), y \geq u_{0}(x) \text { and } \alpha_{t}^{i} \geq x \geq \beta_{t}^{i}\right\} .
$$

Then by definition we have $\mathcal{S} \subset \overline{\Omega_{0}}$. It is also easily seen that $\mathcal{S}$ is convex. On the other hand, Lemma 3.3 and Lemma 2.2 implies that $\overline{\Omega_{t}^{i}} \subset \mathcal{S}$. Hence the convex hull of $\overline{\Omega_{t}^{i}}$ is contained in $\overline{\Omega_{0}}$. 
Lemma 3.5. (Convexity of moving graph pieces) Assume (A1)-(A3). Let $u$ be the solution of (1.2). For any $i=1,2, \ldots, m$ and $t \in\left(0, \tau^{*}\right)$, let $\alpha_{t}^{i}$ and $\beta_{t}^{i}$ be defined as in (3.3). Then

$$
u\left(k x_{1}+(1-k) x_{2}, t\right) \leq k u\left(x_{1}, t\right)+(1-k) u\left(x_{2}, t\right) \quad \text { for any } k \in[0,1],
$$

provided that $x_{1}, x_{2} \in\left[\alpha_{t}^{i}, \beta_{t}^{i}\right]$.

Proof. Set for any $x \in\left[x_{1}, x_{2}\right]$ and $s \in[0, t]$,

$$
v(x, s):=u\left(x_{2}, s\right) \frac{x-x_{1}}{x_{2}-x_{1}}+u\left(x_{1}, s\right) \frac{x_{2}-x}{x_{2}-x_{1}} .
$$

It follows from Lemma 3.4 that $v(x, 0) \geq u_{0}(x)$. It is also obvious that $v\left(x_{1}, s\right)=u\left(x_{1}, s\right)$ and $v\left(x_{2}, s\right)=u\left(x_{2}, s\right)$ for all $s \in[0, t]$. Moreover, due to Lemma 2.2 , we obtain $v_{s}(x, s) \geq 0$ in the viscosity sense for any $x \in\left[x_{1}, x_{2}\right]$ and $s \in(0, t)$. Since $v$ is linear in space variable, the observations above yield that $v$ is a supersolution of (3.1) with $\lambda=t$. Therefore by comparison principle, we have $v(x, t) \geq u(x, t)$ for all $x \in\left[x_{1}, x_{2}\right]$. Our conclusion immediately follows.

Remark 3.5. We remark that the statement in Lemma 3.5 is true for $t=\tau^{*}$ by continuity of the solution $u$.

\subsection{Local bounds for the motion of inflection points}

Our first goal is to show the strict expansion of each convex piece for all $t \in\left[0, \tau^{*}\right)$. To this end, we need to give bounds for $\alpha_{t}^{i}$ and $\beta_{t}^{i}$ for every $i=1,2, \ldots, m$. Roughly speaking, we will find an interval $\left(a_{i}, b_{i}\right)$ such that $\left(\alpha_{t}^{i}, \beta_{t}^{i}\right) \subset\left(a_{i}, b_{i}\right) \subset\left[\beta_{0}^{i-1}, \alpha_{0}^{i+1}\right]$ and the slopes of the tangents to $u_{0}$ in $\left(a_{i}, \alpha_{0}^{i}\right]$ are greater than those in $\left[\beta_{t}^{i}, b_{i}\right)$ for $t \leq \tau^{*}$. To be more precise, let us follow the procedure below to get $\left(a_{i}, b_{i}\right)$ for any fixed $i=1,2, \ldots, m$.

Step 1. We start with the following obstacle problem:

$$
\begin{aligned}
& \min f \in C\left(\left[\beta_{0}^{i-1}, \alpha_{0}^{i+1}\right]\right) \text { subject to } \\
& \qquad\left\{\begin{array}{l}
f^{\prime \prime} \leq 0 \text { in }\left(\beta_{0}^{i-1}, \alpha_{0}^{i+1}\right) \text { in the viscosity sense, } \\
f \geq u_{0} \text { in }\left(\beta_{0}^{i-1}, \alpha_{0}^{i+1}\right) .
\end{array}\right.
\end{aligned}
$$

Owing to (A3), we can easily find a smooth concave function $f \in C^{2}\left(\left[\beta_{0}^{i-1}\right.\right.$, $\left.\left.\alpha_{0}^{i+1}\right]\right)$ satisfying $f \geq u_{0}$ in $\left(\beta_{0}^{i-1}, \alpha_{0}^{i+1}\right), f\left(\beta_{0}^{i-1}\right)=u_{0}\left(\beta_{0}^{i-1}\right)$ and $f\left(\alpha_{0}^{i+1}\right)=$ $u_{0}\left(\alpha_{0}^{i+1}\right)$. Applying Perron's method, we can get a unique solution $\bar{u}_{0}^{i} \in$ $C\left(\left[\beta_{0}^{i-1}, \alpha_{0}^{i+1}\right]\right)$ of $(3.8)$, which is concave in $\left(\beta_{0}^{i-1}, \alpha_{0}^{i+1}\right)$ and satisfies that $\bar{u}_{0}^{i}\left(\beta_{0}^{i-1}\right)=u_{0}\left(\beta_{0}^{i-1}\right)$ and $\bar{u}_{0}^{i}\left(\alpha_{0}^{i+1}\right)=u_{0}\left(\alpha_{0}^{i+1}\right)$. In addition, $\bar{u}_{0}^{i}$ is harmonic at $x \in\left(\beta_{0}^{i-1}, \alpha_{0}^{i+1}\right)$ where $\bar{u}_{0}(x) \neq u_{0}(x)$.

Step 2. Take

$$
\begin{aligned}
& a_{i}=\sup \left\{\xi \in\left(\beta_{0}^{i-1}, \alpha_{0}^{i+1}\right): \bar{u}_{0}^{i}(x)=u_{0}(x) \text { for all } x \in\left(\beta_{0}^{i-1}, \xi\right)\right\}, \\
& b_{i}=\inf \left\{\xi \in\left(\beta_{0}^{i-1}, \alpha_{0}^{i+1}\right): \bar{u}_{0}^{i}(x)=u_{0}(x) \text { for all } x \in\left(\xi, \alpha_{0}^{i+1}\right)\right\} .
\end{aligned}
$$

It is obvious that $a_{i} \leq \alpha_{t}^{i}<\beta_{t}^{i} \leq b_{i}$ by definitions. By the assumption (A3)(e), we have $a_{i}<\alpha_{0}^{i}$ and $b_{i}>\beta_{0}^{i}$. It follows that $\bar{u}_{0}^{i}>u_{0}$ in $\left(a_{i}, b_{i}\right)$, which implies that 


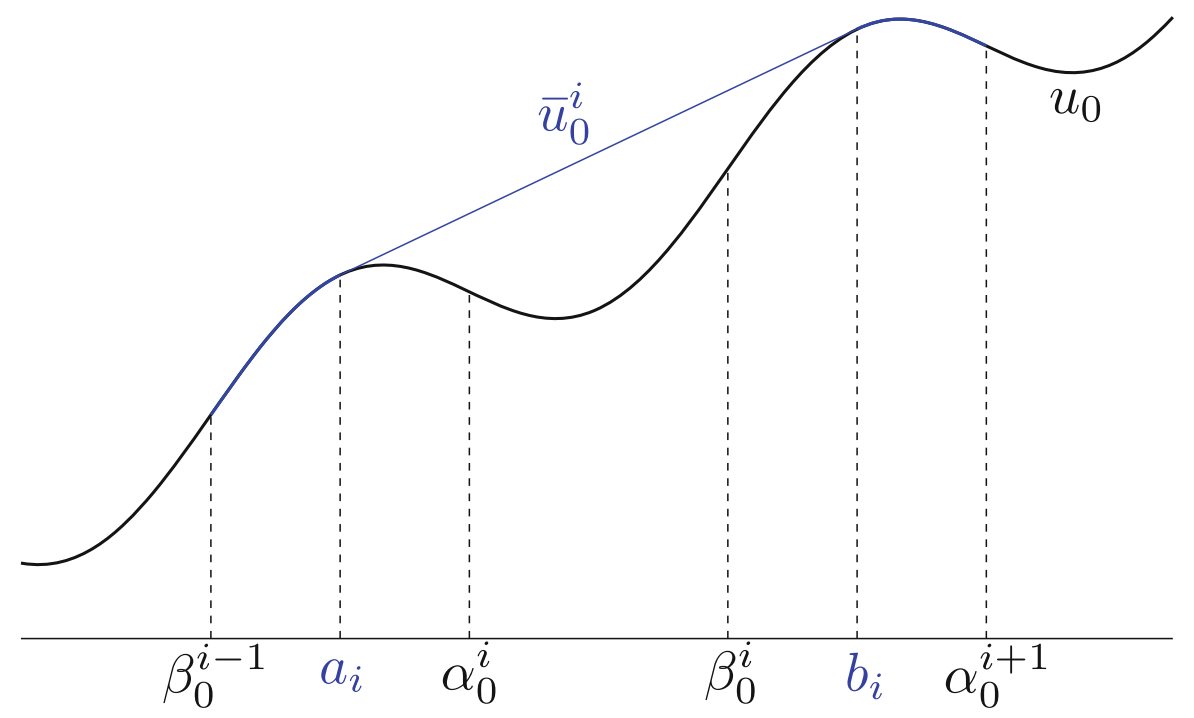

Figure 2. Plot of $\bar{u}_{0}^{i}, a_{i}$ and $b_{i}$

$\bar{u}_{0}^{i}$ is a straight line on $\left(a_{i}, b_{i}\right)$ with slope $k_{i}:=\frac{u_{0}\left(b_{i}\right)-u_{0}\left(a_{i}\right)}{b_{i}-a_{i}}$.

See Fig. 2 for a sketch of $a_{i}, b_{i}$ and $\bar{u}_{0}^{i}$.

Proposition 3.6. (Slope bounds for convex pieces) Assume (A1)-(A3). Let $a_{i}, b_{i}$ be given as in (3.9)-(3.10) and $\bar{u}_{0}^{i}$ be the solution of (3.8) for every $i=1,2, \ldots, m$. Then

$$
\lim _{x \rightarrow a_{i}+} u_{0}^{\prime}(x) \leq \lim _{x \rightarrow b_{i}-} u_{0}^{\prime}(x) .
$$

Remark 3.6. The inequality (3.12) reduces to $u_{0}^{\prime}\left(a_{i}\right) \leq u_{0}^{\prime}\left(b_{i}\right)$ if $a_{i}$ and $b_{i}$ are known to appear in $\left(\beta_{0}^{i-1}, \alpha_{0}^{i+1}\right)$. We keep the weak form (3.12) because it is possible that $a_{i}=\beta_{0}^{i-1}$ or $b_{i}=\alpha_{0}^{i+1}$ and we do not make any further assumptions on the regularity of $u_{0}$ at the inflection points.

Proof. Recall that $u_{0}$ is of class $C^{1}$ in $\left(a_{i}, \alpha_{0}^{i}\right) \cup\left(\beta_{0}^{i}, b_{i}\right)$. The relation (3.12) follows easily, since the graph of $\bar{u}_{0}^{i}$ is a straight line segment above $u_{0}$ in $\left[a_{i}, b_{i}\right]$ with $\bar{u}_{0}^{i}\left(a_{i}\right)=u_{0}\left(a_{i}\right)$ and $\bar{u}_{0}^{i}\left(b_{i}\right)=u_{0}\left(b_{i}\right)$.

The function $\bar{u}_{0}^{i}$ is important in that it plays the role of the asymptotic profile of (1.2) as $t \rightarrow \infty$ provided that each convex piece evolves without being influenced by the others.

Lemma 3.7. (Local large-time behavior) Assume (A1)-(A3). Let $a_{i}, b_{i}$ be given as in (3.9)-(3.10) and $\bar{u}_{0}^{i}$ be the solution of (3.8) for every $i=1,2, \ldots, m$. Let $v(x, t)$ be the solution of

$$
\left\{\begin{array}{l}
v_{t}-h\left(v_{x}\right)\left(v_{x x}\right)_{+}=0 \quad \text { in }\left(a_{i}, b_{i}\right) \times(0, \lambda), \\
v\left(a_{i}, t\right)=u_{0}\left(a_{i}\right) \text { and } v\left(b_{i}, t\right)=u_{0}\left(b_{i}\right) \quad \text { for all } t \in[0, \lambda), \\
v(x, 0)=u_{0}(x) \quad \text { for all } x \in\left[a_{i}, b_{i}\right]
\end{array}\right.
$$


with $\lambda=\infty$. Then $v(x, t) \rightarrow \bar{u}_{0}^{i}(x)$ uniformly for $x \in\left[a_{i}, b_{i}\right]$ as $t \rightarrow \infty$. Moreover,

$$
v(x, t)<\bar{u}_{0}^{i}(x, t) \text { for all }(x, t) \in\left(a_{i}, b_{i}\right) \times[0, \infty) .
$$

Proof. Suppose that the slope of the affine function $\bar{u}_{0}^{i}$ is $k$. Set

$$
\tilde{v}(x, t):=v(x, t)-k\left(x-a_{i}\right) .
$$

Then $\tilde{v}$ is the solution of

$$
\left\{\begin{array}{l}
\tilde{v}_{t}-h\left(\tilde{v}_{x}+k\right)\left(\tilde{v}_{x x}\right)_{+}=0 \quad \text { in }\left(a_{i}, b_{i}\right) \times(0, \infty), \\
\tilde{v}\left(a_{i}, t\right)=u_{0}\left(a_{i}\right) \text { and } \tilde{v}\left(b_{i}, t\right)=u_{0}\left(a_{i}\right) \text { for all } t \in[0, \infty), \\
\tilde{v}(x, 0)=u_{0}(x)-k\left(x-a_{i}\right) \text { for all } x \in\left[a_{i}, b_{i}\right]
\end{array}\right.
$$

Noticing that $u_{0}\left(a_{i}\right)$ is the unique stationary solution of the equation above, we may apply the same argument as in the proof of Proposition 2.3 to get that

$$
\tilde{v}(x, t) \rightarrow u_{0}\left(a_{i}\right) \text { uniformly for all } x \in\left[a_{i}, b_{i}\right] \text { as } t \rightarrow \infty,
$$

which amounts to saying that $v(\cdot, t) \rightarrow \bar{u}_{0}^{i}(\cdot)$ in $\left[a_{i}, b_{i}\right]$.

We next show (3.14). We first show that for any $t \geq 0$,

$$
\min _{x \in\left(a_{i}, b_{i}\right)} \tilde{v}(x, t)<u_{0}\left(a_{i}\right)
$$

Let us construct a supersolution of (1.2). Let $W_{0}(x)=u_{0}\left(a_{i}\right)+A \sin (\omega x+d)$ with $A, \omega$ and $d$ properly chosen such that $\left[a_{i}, b_{i}\right]$ is a period of $W_{0}$ with

$$
W_{0}\left(a_{i}\right)=W_{0}\left(b_{i}\right)=\max _{\mathbb{R}} W_{0}
$$

and $W_{0}(x)>u_{0}(x)-k\left(x-a_{i}\right)$ for all $x \in\left[a_{i}, b_{i}\right]$. We solve the heat equation

$$
W_{t}-C W_{x x}=0 \quad \text { in } \mathbb{R} \times(0, \infty)
$$

with $C>0$ and $W(x, 0)=W_{0}(x)$. The unique solution of the Cauchy problem is $W(x, t)=u_{0}\left(a_{i}\right)+A e^{-C \omega^{2} t} \sin (\omega x+d)$. We thus have $W\left(a_{i}, t\right)=W\left(b_{i}, t\right)>$ $u_{0}\left(a_{i}\right)$ for all $t \geq 0$. In addition, $W(x, t)$ is convex in $x$ whenever $W(x, t)<$ $u_{0}\left(a_{i}\right)$. Since $W \equiv u_{0}\left(a_{i}\right)$ is clearly another solution of (3.17),

$$
\bar{W}(x, t)=\min \left\{W(x, t), u_{0}\left(a_{i}\right)\right\}
$$

is a supersolution of (3.17).

We next claim that $\bar{W}$ restricted in $\left[a_{i}, b_{i}\right] \times[0, \infty)$ is also a supersolution of (3.15) provided that $C>0$ is large enough. Indeed, the boundary conditions are clearly satisfied. Also, by calculation, we have

$$
\sup _{(x, t) \in \mathbb{R} \times(0, \infty)}\left|W_{x}(x, t)\right| \leq|A \omega| \quad \text { for any } C>0 .
$$

Now letting $C \geq \max _{|p| \leq|A \omega|} h(p+k)$, we have

$$
W_{t}-h\left(W_{x}\right) W_{x x} \geq W_{t}-C W_{x x} \text { provided that } W_{x x} \geq 0 .
$$

It follows that $\bar{W}(x, t)$ is a supersolution of (3.15) in the region where $\bar{W}<u_{0}\left(a_{i}\right)$. Besides, it is easy to see that $\bar{W}(x, t)$ satisfies the definition of supersolutions for $(x, t) \in\left(a_{i}, b_{i}\right) \times(0, \infty)$ with $W(x, t)>u_{0}\left(a_{i}\right)$ because $\bar{W}$ is just a constant around those points. Our last verification is for those $(x, t)$ satisfying $W(x, t)=u_{0}\left(a_{i}\right)$ but in fact $\bar{W}$ cannot be tested from below there. 
By comparison principle for (3.15), we obtain $\tilde{v}(x, t) \leq \bar{W}(x, t)$ and (3.16) follows immediately. Noticing that $\bar{u}_{0}^{i}(x)>v(x, t)$ in $\left(a_{i}, \alpha_{t}^{i}\right] \cup\left[\beta_{t}^{i}, b_{i}\right)$ due to $(\mathrm{A} 3)(\mathrm{c})$ and the convexity of $u$ in $\left[\alpha_{t}^{i}, \beta_{t}^{i}\right]$, we easily deduce (3.14) from $(3.16)$.

The argument above can be adapted to the proof of the following general result.

Theorem 3.8. Suppose $v$ is the viscosity solution of

$$
\left\{\begin{array}{l}
v_{t}-h\left(v_{x}\right)\left(v_{x x}\right)_{+}=0 \quad \text { in }\left(a_{i}, b_{i}\right) \times(0, T), \\
v\left(x_{1}, t\right)=u_{0}\left(x_{1}\right) \text { and } v\left(x_{2}, t\right)=u_{0}\left(x_{2}\right) \quad \text { for all } t \in[0, \infty), \\
v(x, 0)=u_{0}(x) \quad \text { for all } x \in\left[x_{1}, x_{2}\right]
\end{array}\right.
$$

with

$u_{0}(x)<\bar{u}_{0}(x)=\frac{\left(x_{2}-x\right) u_{0}\left(x_{1}\right)+\left(x-x_{1}\right) u_{0}\left(x_{2}\right)}{x_{2}-x_{1}} \quad$ for any $x \in\left(x_{1}, x_{2}\right)$.

Then $v(x, t) \rightarrow \bar{u}_{0}(x)$ as $t \rightarrow \infty$ uniformly in $x \in\left[x_{1}, x_{2}\right]$. Moreover,

$$
v(x, t)<\bar{u}(x) \quad \text { for every }(x, t) \in\left(x_{1}, x_{2}\right) \times[0, \infty) .
$$

Lemma 3.7 asserts that an independent convex piece will eventually become a straight line and this asymptotic state is realized only at $t=\infty$. We next utilize this fact to deduce the following key result. For any $t \in\left(0, \tau^{*}\right]$, we set

$k_{t}^{\alpha, i}:=\lim _{x \rightarrow \alpha_{t}^{i}-} \frac{u(x, t)-u\left(\alpha_{t}^{i}, t\right)}{x-\alpha_{t}^{i}} \quad$ and $\quad k_{t}^{\beta, i}:=\lim _{x \rightarrow \beta_{t}^{i}+} \frac{u(x, t)-u_{0}\left(\beta_{t}^{i}\right)}{x-\beta_{t}^{i}}$.

Lemma 3.9. (Strict upper bounds of convex pieces) Assume (A1)-(A3). Let $u$ be the solution of (1.2). Let $\alpha_{t}^{i}, \beta_{t}^{i}$ be defined as in (3.3) and $\bar{u}_{0}^{i}$ be defined as in (3.8). Then

$$
u(x, t)<\bar{u}_{0}^{i}(x) \quad \text { for any }(x, t) \in\left(\alpha_{t}^{i}, \beta_{t}^{i}\right) \times\left[0, \tau^{*}\right)
$$

and

$$
k_{t}^{\alpha, i}<k_{t}^{\beta, i},
$$

for any $i=1,2, \ldots, m$ and $t \in\left[0, \tau^{*}\right)$, where $k_{t}^{\alpha, i}$ and $k_{t}^{\beta, i}$ is given in (3.18).

Proof. We first note that (3.19) is a straightforward consequence of Lemma 3.7, since $u$ restricted for $t \in\left[0, \tau^{*}\right)$ is the solution of (3.13) with $\lambda=\tau^{*}$. To show (3.20), let us first discuss the case that $\tau^{*}=\infty$. As mentioned in Remark 3.1, there are $x_{i} \in\left[\beta_{0}^{i}, \alpha_{0}^{i+1}\right]$ such that $u_{0}\left(x_{i}\right)=M=\max _{x \in I} u_{0}(x)$ for each $i=0,1, \ldots, m$. Lemma 2.2 yields that $u\left(x_{i}, t\right)=M$ for all $t \geq 0$, which implies that $\alpha_{t}^{i}, \beta_{t}^{i} \in\left[x_{i}, x_{i+1}\right]$ for any $t \geq 0$ and $x_{i-1} \leq a_{i}<b_{i} \leq x_{i}$ with $i=1,2, \ldots, m$. We next show that in fact $\alpha_{t}^{i}, \beta_{t}^{i} \in\left(x_{i-1}, x_{i}\right)$. If it is not the case, say $\alpha_{t}^{i}=x_{i-1}$ for some fixed $t \geq 0$ and $i=0,1, \ldots, m$, then by Lemma 3.3, the graph of $u(x, t)$ is above the tangent line to the graph of $u_{0}$ at $\alpha_{t}^{i}=x_{0}$, which is exactly $y=M$, for all $x \in\left[\alpha_{t}^{i}, \beta_{t}^{i}\right]$. It follows that $u(x, t) \equiv M$ 
for all $x \in\left[a_{i}, b_{i}\right]$, which contradicts Lemma 3.7. By the strict concavity of $u_{0}$ in $\left[x_{i-1}, \alpha_{0}^{i}\right)$ and in $\left(\beta_{0}^{i}, x_{i}\right]$, we immediately get

$$
k_{t}^{\alpha, i}<0=u_{0}^{\prime}\left(x_{i-1}\right)=u_{0}^{\prime}\left(x_{i}\right)<k_{t}^{\beta, i} \text { for all } t \geq 0 .
$$

We then discuss the case $\tau^{*}<\infty$. Let us show that either $\alpha_{t}^{i}>a_{i}$ or $\beta_{t}^{i}<b_{i}$ for all $t \in\left[0, \tau^{*}\right)$. We again argue by contradiction. Suppose on the contrary that $\alpha_{t}^{i}=a_{i}$ and $\beta_{t}^{i}=b_{i}$. As in the proof of Proposition 3.6, the slope $k_{i}$ of $\bar{u}_{0}^{i}$ in $\left(a_{i}, b_{i}\right)$ is equal to either $u_{0}^{\prime}\left(a_{i}\right)$ or $u_{0}^{\prime}\left(b_{i}\right)$, which gives $u(x, t) \geq \bar{u}_{0}^{i}$ in $\left(a_{i}, b_{i}\right)$ by Lemma 3.3. This is a contradiction to Lemma 3.7, since $u$ can be viewed as the solution of (3.13) for any $i$ when $t<\tau^{*}$.

When $\alpha_{t}^{i}>a_{i}$ and $\beta_{t}^{i}<b_{i}$, it is easy to get (3.20), again due to the strict concavity of $u_{0}$ in $\left[a_{i}, \alpha_{0}^{i}\right) \cup\left(\beta_{0}^{i}, b_{i}\right]$. Suppose $\alpha_{t}^{i}>a_{i}$ and $\beta_{t}^{i}=b_{i}$. Then $b_{i}<\alpha_{0}^{i+1}$ for otherwise it contradicts the fact that $t<t^{*}$. This means that $k_{t}^{\beta, i}=u_{0}^{\prime}\left(b_{i}\right)$. On the other hand, the concavity of $u_{0}$ yields $k_{t}^{\alpha, i}<$ $\lim _{x \rightarrow a_{i}}+u_{0}^{\prime}(x)$. Therefore (3.20) follows easily from Proposition 3.6. The other case that $\alpha_{t}^{i}=a_{i}$ and $\beta_{t}^{i}<b_{i}$ is similarly treated.

Remark 3.7. With slight modification in the proof above, we may also prove that

$$
u\left(\cdot, \tau^{*}\right)<\bar{u}_{0}^{i}(\cdot) \quad \text { in }\left(\alpha_{\tau^{*}}^{i}, \beta_{\tau^{*}}^{i}\right)
$$

and

$$
k_{\tau^{*}}^{\alpha, i}<k_{\tau^{*}}^{\beta, i}
$$

for all $i=1,2, \ldots, m$ provided that $\tau^{*}<\infty$. Indeed, Lemma 3.3 and Lemma 3.7 still enable us to deduce that either $\alpha_{\tau^{*}}^{i}>a_{i}$ or $\beta_{\tau^{*}}^{i}<b_{i}$ in this case. The proof is exactly the same as that of Theorem 3.20 if $\alpha_{\tau^{*}}^{i}>a_{i}$ and $\beta_{\tau^{*}}^{i}<b_{i}$.

We therefore assume, for instance, that $\alpha_{\tau^{*}}^{i}>a_{i}$ and $\beta_{\tau^{*}}^{i}=b_{i}$. We are not able to exclude the possibility that $b_{i}=\alpha_{0}^{i+1}$ this time but we may instead use Lemma 2.2 and $(\mathrm{A} 3)(\mathrm{d})$ to show that $k_{\tau^{*}}^{\beta, i} \geq k_{0}^{\alpha, i+1}=\lim _{x \rightarrow b_{i}-} u_{0}^{\prime}(x)$, which, combined with Proposition 3.6, implies (3.22). The strict upper boundedness (3.21) is also easily shown, since $u\left(\alpha_{\tau^{*}}^{i}, \tau^{*}\right)<\bar{u}_{0}^{i}\left(\alpha_{\tau^{*}}^{i}\right)$ and $u\left(\cdot, \tau^{*}\right)$ is convex in $\left[\alpha_{\tau^{*}}^{i}, \beta_{\tau^{*}}^{i}\right]$, as mentioned in Remark 3.5.

Remark 3.8. By Lemma 3.9 and Remark 3.7, we must have $\alpha_{t}^{1}>\beta_{t}^{0} \equiv a$ and $\beta_{t}^{m}<\alpha_{t}^{m+1}=b$ either for all $t \in[0, \infty)$ if $\tau^{*}=\infty$ or for all $t \in\left[0, \tau^{*}\right]$ if $\tau^{*}<\infty$.

\subsection{Short time behavior of the graph}

Lemma 3.10. (Immediate move of a corner) Assume (A1). Assume that $u_{0} \in$ $C(\mathbb{R})$ is periodic. Let $u$ be the solution of (1.2) with initial data $u_{0}$. If there is $x_{0} \in \mathbb{R}$ such that

$$
-\infty<\lim _{x \rightarrow x_{0}-} \frac{u_{0}(x)-u_{0}\left(x_{0}\right)}{x-x_{0}}<\lim _{x \rightarrow x_{0}+} \frac{u_{0}(x)-u_{0}\left(x_{0}\right)}{x-x_{0}}<\infty,
$$

then $u\left(x_{0}, t\right)>u_{0}\left(x_{0}\right)$ for any $t>0$. 
Proof. Assume by contradiction that there is $t_{0}>0$ such that $u\left(x_{0}, t\right)=u_{0}\left(x_{0}\right)$ for all $t \in\left[0, t_{0}\right]$. Since $u(x, t)$ is nondecreasing in $t$, by the assumptions given, it is easy to construct a smooth periodic function $w: \mathbb{R} \times[0, \delta]$ such that

(1) $w(x, t)=w_{0}(x)$ for all $x \in \mathbb{R}$ and $t \in\left[0, t_{0}\right]$;

(2) $w_{0}(x) \leq u_{0}(x)$ for all $x \in \mathbb{R}$ and $w_{0}\left(x_{0}\right)=u_{0}\left(x_{0}\right)$;

(3) $w_{0}^{\prime \prime}\left(x_{0}\right)>0$.

Then it follows that $w$ satisfies

$$
w_{t}\left(x_{0}, t\right)-h\left(w_{x}\left(x_{0}, t\right)\right)\left(w_{x x}\left(x_{0}, t\right)\right)_{+}<0 \quad \text { for all } t \in\left(0, t_{0}\right),
$$

which contradicts the fact that $u(x, t)$ is a solution of $(1.2)$ as $w(x, t)$ touches $u(x, t)$ from below at the point $\left(x_{0}, t\right)$ with $t$ arbitrarily taken from $\left(0, t_{0}\right)$.

The argument in the proof above can also be generalized to show a similar situation during the evolution.

Lemma 3.11. If $u(x, t)$ is a continuous and space-periodic solution of (1.2) satisfying

$$
-\infty<\lim _{x \rightarrow x_{0}-} \frac{u\left(x, t_{0}\right)-u\left(x_{0}, t_{0}\right)}{x-x_{0}}<\lim _{x \rightarrow x_{0}+} \frac{u\left(x, t_{0}\right)-u\left(x_{0}, t_{0}\right)}{x-x_{0}}<\infty
$$

for some $x_{0} \in \mathbb{R}$ and $t_{0} \geq 0$. Then $u\left(x_{0}, t\right)>u\left(x_{0}, t_{0}\right)$ for all $t>t_{0}$.

Let $\tilde{u}(x, t)=u\left(x, t+t_{0}\right)$. Then $\tilde{u}$ is the solution of (1.3) with initial data $\tilde{u}(x, 0)=u\left(x, t_{0}\right)$. We reach the conclusion by applying Lemma 3.10.

Theorem 3.12. (Nonstop moving of convex pieces) Assume (A1)-(A3). Let $u$ be the solution of (1.2) and $\tau^{*}$ be given as in (3.4). Then $u(x, t)>u(x, s)$ for all $x \in\left[\alpha_{s}^{i}, \beta_{s}^{i}\right]$ and $0 \leq s<t<\tau^{*}$.

Proof. Suppose by contradiction that there are $s \geq 0, \delta>0$ and $x_{0} \in\left[\alpha_{s}^{i}, \beta_{s}^{i}\right]$ such that $u\left(x_{0}, t\right)=u\left(x_{0}, s\right)$ for all $t \in[s, s+\delta]$. By Lemma 3.5, we have

$$
-\infty<\lim _{x \rightarrow x_{0}-} \frac{u(x, s)-u\left(x_{0}, s\right)}{x-x_{0}} \leq \lim _{x \rightarrow x_{0}+} \frac{u(x, s)-u\left(x_{0}, s\right)}{x-x_{0}}<\infty .
$$

We may derive a contradiction immediately by using Lemma 3.11 if

$$
\lim _{x \rightarrow x_{0}-} \frac{u(x, s)-u\left(x_{0}, s\right)}{x-x_{0}}<\lim _{x \rightarrow x_{0}+} \frac{u(x, s)-u\left(x_{0}, s\right)}{x-x_{0}} .
$$

We therefore only discuss the case that

$$
\lim _{x \rightarrow x_{0}-} \frac{u(x, s)-u\left(x_{0}, s\right)}{x-x_{0}}=\lim _{x \rightarrow x_{0}+} \frac{u(x, s)-u\left(x_{0}, s\right)}{x-x_{0}},
$$

which means $u(\cdot, s)$ is differentiable at $x_{0}$. Let us denote by $\hat{k}$ the quantity above, i.e., the derivative at $x_{0}$. By Lemma 3.3 and Lemma 3.5, we have $u_{0}^{\prime}\left(\alpha_{s}^{i}\right) \leq \hat{k} \leq u_{0}^{\prime}\left(\beta_{s}^{i}\right)$. Since Lemma 3.9 states that $k_{s}^{\alpha, i}<k_{s}^{\beta, i}$, we must have either $\hat{k}>k_{s}^{\alpha, i}$ or $\hat{k}<k_{s}^{\beta, i}$. Let us assume the latter without loss of generality. Note that in this case, we may take a smooth periodic function $w_{0}$ which fulfills the following conditions: 
(1) Its graph is symmetric about $\left(x_{0}, u_{0}\left(x_{0}\right)\right)$, i.e., $w_{0}(x)=2 u_{0}\left(x_{0}\right)-w_{0}\left(2 x_{0}-\right.$ $x)$.

(2) It serves as a one-sided lower bound in the sense that $w_{0}(x)=\hat{k} x$ for all $x \in\left[x_{0}, x_{0}+\delta_{1}\right)$ and with some $\delta_{1}>0$ and $w_{0}(x)<u(x, s)$ for all $x \in\left(\beta_{s}^{i}, \infty\right)$.

(3) Its first derivative is nondecreasing in $\left[x_{0}, \beta_{s}^{i}\right]$ and

$$
w_{0}^{\prime}(x) \equiv \bar{k}>\hat{k} \quad \text { for all } x \in\left(\beta_{s}^{i}, \infty\right) .
$$

Set $K=\sup _{x}\left|w_{0}^{\prime}(x)\right|$ and $c=\min _{|p| \leq K} h(p)$. It is clear that $c>0$ due to (A1). Let $w$ be the unique smooth solution of a heat equation

$$
\begin{cases}w_{t}-c w_{x x}=0 & \text { in } \mathbb{R} \times(s, \infty), \\ w(x, s)=w_{0}(x) & \text { in } \mathbb{R} .\end{cases}
$$

We then utilize the symmetry to get $w\left(x_{0}, t\right)=u_{0}\left(x_{0}\right)$ and $w_{x x}\left(x_{0}, t\right)=0$ for all $t \geq s$. We adopt the strong maximum principle for $w_{x}$ to obtain $w_{x}\left(x_{0}, t\right)>$ $w_{0}^{\prime}\left(x_{0}\right)$ for $t \geq s$ and therefore

$$
w_{x}\left(x_{0}, t\right)>\hat{k}=\lim _{x \rightarrow x_{0}+} \frac{u(x, t)-u\left(x_{0}, t\right)}{x-x_{0}} \text { for all } t>s .
$$

Moreover, the maximum principle for $w_{x x}$ implies that $w_{x x} \geq 0$ in $\left(x_{0}, \infty\right) \times$ $[s, s+\delta)$. Besides, there exists $\mu \in(0, \delta)$ small such that $w\left(\beta_{s}^{i}, t\right) \leq u\left(\beta_{s}^{i}, t\right)$ for all $t \in[s, s+\mu]$.

It is not difficult to see that $w$ also satisfies

$$
\begin{cases}w_{t}-h\left(w_{x}\right)\left(w_{x x}\right)_{+} \leq 0 & \text { in }\left(x_{0}, \beta_{s}^{i}\right) \times(s, s+\mu), \\ w(x, s) \leq u_{0}(x) & \text { for all } x \in\left[x_{0}, \beta_{s}^{i}\right], \\ w\left(x_{0}, t\right) \leq u\left(x_{0}, t\right) \text { and } w\left(\beta_{s}^{i}, t\right) \leq u\left(\beta_{s}^{i}, t\right) & \text { for all } t \in[s, s+\mu] .\end{cases}
$$

Indeed, since the initial and boundary conditions are satisfied, as proved above, we only need to show that $w$ fulfills the first inequality. Note that the maximum principle for (3.24) gives

$$
\left|w_{x}(x, t)\right|<K \quad \text { for all } x \in \mathbb{R} \text { and } t \geq 0 .
$$

Since $w$ stays convex in space in $\left(x_{0}, \infty\right) \times(s, s+\mu)$. Then a direct calculation shows that

$$
w_{t}=c w_{x x} \leq h\left(w_{x}\right)\left(w_{x x}\right)_{+} \quad \text { in }\left(x_{0}, \beta_{s}^{i}\right) \times(s, s+\mu),
$$

which completes the verification that $w$ fulfills (3.26). As a result, $u(x, t) \geq$ $w(x, t)$ for all $x \in\left[x_{0}, \beta_{s}^{i}\right]$ and $t \in[s, s+\mu)$, which in turn implies that

$$
\lim _{x \rightarrow x_{0}+} \frac{u(x, t)-u\left(x_{0}, t\right)}{x-x_{0}} \geq w_{x}\left(x_{0}, t\right)>\hat{k}=\lim _{x \rightarrow x_{0}-} \frac{u(x, t)-u\left(x_{0}, t\right)}{x-x_{0}}
$$

for any $t \in(s, s+\mu)$. In particular, the relation above holds for $t=s+\mu$. Since $\mu<\delta$, Lemma 3.11 now comes into play for us to obtain $u\left(x_{0}, s+\delta\right)>u\left(x_{0}, s\right)$, which is a contradiction.

We conclude the proof by pointing out that a symmetric version of the argument above applies to the case when $\hat{k}>k_{s}^{\alpha, i}$. 
Corollary 3.13. (Short time behavior of inflection points) Assume (A1)-(A3). Then for any $i=1,2, \ldots, m$ and $0 \leq s<t<\tau^{*}$, we have $\alpha_{t}^{i}<\alpha_{s}^{i}$ and $\beta_{t}^{i}>\beta_{s}^{i}$.

Proof. We only show $\beta_{t}^{i}>\beta_{s}^{i}$. By Lemma 2.2 , it is not difficult to find that every moving piece, which is defined on $\left[\alpha_{t}^{i}, \beta_{t}^{i}\right]$, is expanding; in other words, we have $\alpha_{t}^{i} \leq \alpha_{s}^{i}$ and $\beta_{t}^{i} \geq \beta_{s}^{i}$ for all $t>s \geq 0$. It suffices to show that $\beta_{t}^{i} \neq \beta_{s}^{i}$ but this is a straightforward consequence of Theorem 3.12 and the continuity of the solution $u$.

\subsection{Global behavior of the graph}

In the previous section we have given a rigorous analysis for the motion of the graph and inflection points before the first collision time $\tau^{*}$. We are interested in the evolution after $\tau^{*}$ in what follows. Our basic idea is to regard the solution $u(x, t)$ as a new initial condition every time when two inflection points hit each other and apply the preceding results repeatedly.

To be more precise, we investigate the shape of the graph of $u(x, t)$ at $t=\tau^{*}<\infty$ below.

Lemma 3.14. Assume (A1)-(A3). Let $u$ be the solution of (1.2) and $\tau^{*}$ be defined as in (3.4). Assume $\tau^{*}<\infty$. Then there exists $I_{0}^{*}=\cup_{i=1}^{m^{*}}\left\{\bar{\alpha}_{0}^{i}, \bar{\beta}_{0}^{i}\right\}$, where $m^{*} \in \mathbb{Z}$ with $1 \leq m^{*}<m$ and $\bar{\alpha}_{0}^{i}, \bar{\beta}_{0}^{i} \in I$ such that

(1) $\bar{\alpha}_{0}^{i}<\bar{\beta}_{0}^{i}<\bar{\alpha}_{0}^{i+1}<\bar{\beta}_{0}^{i+1}$ for all $i=1,2, \ldots, m^{*}-1$;

(2) $u\left(\cdot, \tau^{*}\right)$ is convex in $I_{+}^{*}:=\cup_{i=1}^{m^{*}}\left(\bar{\alpha}_{0}^{i}, \bar{\beta}_{0}^{i}\right)$;

(3) $u\left(\cdot, \tau^{*}\right)$ is of class $C^{1}$ in $I_{-}^{*}:=I \backslash\left(I_{+}^{*} \cup I_{0}^{*}\right)$ with $x \mapsto u_{x}\left(x, \tau^{*}\right)$ strictly decreasing in each interval of $I_{-}^{*}$;

(4) For any $\bar{\alpha}_{0}^{i}, \bar{\beta}_{0}^{i} \in I_{0}^{*}$,

$$
\begin{aligned}
& \bar{k}_{0}^{\alpha, i}:=\lim _{x \rightarrow \bar{\alpha}_{0}^{i}+} \frac{u_{0}(x)-u_{0}\left(\bar{\alpha}_{0}^{i}\right)}{x-\bar{\alpha}_{0}^{i}} \geq \lim _{x \rightarrow \bar{\alpha}_{0}^{i}-} \frac{u_{0}(x)-u_{0}\left(\bar{\alpha}_{0}^{i}\right)}{x-\bar{\alpha}_{0}^{i}} \quad \text { and } \\
& \bar{k}_{0}^{\beta, i}:=\lim _{x \rightarrow \bar{\beta}_{0}^{i}-} \frac{u_{0}(x)-u_{0}\left(\bar{\beta}_{0}^{i}\right)}{x-\bar{\beta}_{0}^{i}} \leq \lim _{x \rightarrow \bar{\beta}_{0}^{i}+} \frac{u_{0}(x)-u_{0}\left(\bar{\beta}_{0}^{i}\right)}{x-\bar{\beta}_{0}^{i}} .
\end{aligned}
$$

(5) $\bar{k}_{0}^{\alpha, i}<\bar{k}_{0}^{\beta, i}$ for any $i=1,2, \ldots, m^{*}$.

Proof. It follows from Remark 3.3 that there exists $i=0,1, \ldots, m$ such that

$$
\beta_{\tau^{*}}^{i}=\alpha_{\tau^{*}}^{i+1}
$$

Let us take the least $i$, denoted by $i_{1}$, satisfying (3.27) for our explanation. Assume that $i_{j}:=i_{1}+j-1$ also satisfies (3.27) for all $j=1,2, \ldots, l$ with $i_{l} \leq m$. We first claim that $i_{1}>0$ and $i_{l}<m$. If $i_{1}=0$, then $u_{0}^{\prime}(a)=$ $u_{0}^{\prime}\left(\beta_{\tau^{*}}^{0}\right)=u_{0}^{\prime}\left(\alpha_{\tau^{*}}^{1}\right)=0$, which, by Remark 3.4 and Remark 3.5 , yields that $u_{0}^{\prime}\left(\beta_{\tau^{*}}^{1}\right)=0$ too. This is a contradiction to Remark 3.7. We get $i_{l}<m$ for the same reason. We therefore let $\bar{\alpha}_{0}^{i}=\alpha_{\tau^{*}}^{i}$ for all $i=1,2, \ldots, i_{1}, \bar{\beta}_{0}^{i}=\beta_{\tau^{*}}^{i}$ for all $i=1,2, \ldots, i_{1}-1$ and $\bar{\beta}_{0}^{i_{1}}=\beta_{\tau^{*}}^{i_{l}+1}$. We continue relabeling the inflection points in this way if $i_{l}+1<m$ and get $\bar{\alpha}_{0}^{i}$ and $\bar{\beta}_{0}^{i}$ for $i=1,2, \ldots, m^{*}$ with 
$m^{*}<m$. This gives the partition in (1). Also, it is clear that (3) holds, since $I_{-}^{*} \subset I_{-}$and $u\left(x, \tau^{*}\right)=u_{0}(x)$ for all $x \in I_{-}^{*}$. Using Remark 3.4, we conclude (4) with ease.

We next show (2) and (5). By Remark 3.5 and Remark 3.7, we may assert that $u\left(\cdot, \tau^{*}\right)$ is convex in each interval $\left(\alpha_{\tau^{*}}^{i}, \beta_{\tau^{*}}^{i}\right)$ and

$$
k_{\tau^{*}}^{\alpha, i}<k_{\tau^{*}}^{\beta, i}
$$

for all $i=1,2, \ldots, m$. Since $\left(\bar{\alpha}_{0}^{i}, \bar{\beta}_{0}^{i}\right)=\left(\alpha_{\tau^{*}}^{i}, \beta_{\tau^{*}}^{i}\right)$ for all $i=1,2, \ldots, i_{1}-1$, the convexity and the slope relation still hold for these intervals. We thus need to prove them in the interval $\left(\bar{\alpha}_{0}^{i_{1}}, \bar{\beta}_{0}^{i_{1}}\right)=\cup_{j=1}^{l+1}\left(\alpha_{0}^{i_{j}}, \beta_{0}^{i_{j}}\right)$. We adopt Remark 3.4 and obtain

$$
u\left(\cdot, \tau^{*}\right) \geq L_{\tau^{*}}^{\beta, i_{j}} \quad \text { in }\left[\alpha_{\tau^{*}}^{i_{j}}, \beta_{\tau^{*}}^{i_{j}}\right] \quad \text { and } \quad u\left(\cdot, \tau^{*}\right) \geq L_{\tau^{*}}^{\alpha, i_{j}+1} \quad \text { in }\left[\alpha_{\tau^{*}}^{i_{j}+1}, \beta_{\tau^{*}}^{i_{j}+1}\right]
$$

for all $j=1,2, \ldots, l$, from which the convexity follows easily, since $L_{\tau^{*}}^{\beta, i_{j}}=$ $L_{\tau^{*}}^{\alpha, i_{j}+1}$. Finally, we combine the relation $k_{\tau^{*}}^{\beta, i_{j}}=k_{\tau^{*}}^{\alpha, i_{j}+1}$ for $j=1,2, \ldots, l$ with (3.28) and get

$$
\bar{k}_{0}^{\alpha, i_{1}}=k_{\tau^{*}}^{\alpha, i_{1}}<k_{\tau^{*}}^{\beta, i_{1}+l}=\bar{k}_{0}^{\alpha, i_{1}} .
$$

The proof of (2) and (5) for the other inflection points $\bar{\alpha}_{0}^{i}$ and $\bar{\beta}_{0}^{i}$ is analogous.

Lemma 3.14 amounts to saying that $u\left(\cdot, \tau^{*}\right)$ satisfies the initial condition (A3). Since it obviously satisfies (A2) as well, we may discuss the graph motion after $t=\tau^{*}$ by repeating the analysis presented in Sects. 3.2, 3.3 and 3.4 for only finite times.

In order to state our main theorem, we define for any $t \geq 0$

$$
I_{-}^{t}:=\cup_{i=0}^{m}\left(\beta_{t}^{i}, \alpha_{t}^{i+1}\right), \quad I_{0}^{t}=I \cap \partial I_{-}^{t} \quad \text { and } \quad I_{+}^{t}=I \backslash\left(I_{-}^{t} \cup i_{0}^{t}\right) .
$$

Theorem 3.15. (Long term nonstop moving of convex pieces) Assume (A1)(A3). Let $u$ be the solution of (1.2) and $\alpha_{s}^{i}$ and $\beta_{s}^{i}$ be defined for any $s \geq 0$ as in (3.3). Then $u(x, t)>u(x, s)$ for all $t>s \geq 0$ and $x \in I_{+}^{s} \cup I_{0}^{s}$.

Theorem 3.16. (Continuity of waiting time) Assume (A1)-(A3). Then the waiting time $T_{0}(z)$ of the curve $y=u_{0}(x)$ at $z=\left(x, u_{0}(x)\right)$ is continuous with respect to $x \in I$. Moreover, $T_{0}(x) \rightarrow \infty$ as $[a, b] \ni x \rightarrow x_{0}$ when $x_{0}=a$ or $b$.

Proof. To simplify our notation in the proof, we view $T_{0}$ as a function of $x$, i.e., $T_{0}(x):=T_{0}\left(x, u_{0}(x)\right)$. It is clear that $T_{0}(x)=0$ for all $x \in I_{+}$. Let us prove the continuity of $T_{0}$ for any $x_{0} \in I \backslash I_{+}$. Without loss, we assume $x_{0} \in\left[\beta_{0}, b\right)$.

We first show the continuity of $T_{0}$ at any fixed $x \in I$ such that $T_{0}(x) \in$ $\left[0, \tau^{*}\right)$. We have three different cases to discuss.

1. $T_{0}(x)=0$. Then we have $x \in I_{+} \cup I_{0}$ due to Theorem 3.1. In other words, there exists $i=1,2, \ldots, m$ such that $x \in\left[\alpha_{0}^{i}, \beta_{0}^{i}\right]$. By Corollary 3.13, for any $\delta>0$, we have $\left[\alpha_{0}^{i}, \beta_{0}^{i}\right] \subset\left(\alpha_{\delta}^{i}, \beta_{\delta}^{i}\right)$. We are thus allowed to take $r>0$ small such that $(x-r, x+r) \subset\left(\alpha_{\delta}^{i}, \beta_{\delta}^{i}\right)$, which means that $T_{0}(y)<t$ for all $y \in(x-r, x+r)$. 
2. $T_{0}(x)=s \in\left(0, \tau^{*}\right)$. Then $x \neq a$ and $x \neq b$ due to Remark 3.8. We claim there exists $i=1,2, \ldots, m$ such that $x=\alpha_{s}^{i}$ or $x=\beta_{s}^{i}$. Indeed, $x \notin$ $\cup_{i=1}^{m+1}\left(\beta_{s}^{i-1}, \alpha_{s}^{i}\right)$, for otherwise $T_{0}(x)>s$, as a result of Theorem 3.1 applied to the solution of (1.2) with initial data $u(x, s)$. Also, $x \notin \cup_{i=1}^{m}\left(\alpha_{s}^{i}, \beta_{s}^{i}\right)$, since, by Corollary 3.13, $T_{0}(x)<s$ for every $x$ in the set. For any $\delta>0$ small, we use Corollary 3.13 again to get $r>0$ such that $(x-r, x+r) \subset$ $\left(\alpha_{s+\delta}^{i}, \beta_{s+\delta}^{i}\right) \backslash\left(\alpha_{s-\delta}^{i}, \beta_{s-\delta}^{i}\right)$, which implies that $T_{0}(y) \in(s-\delta, s+\delta)$ for any $y \in(x-r, x+r)$.

We extend our argument to the remaining case when $T_{0}(x) \geq \tau^{*}$ by considering the problem with updated initial conditions. In addition, for the endpoints $a$ and $b$, then we may apply Theorem 3.8 with $v=u, x_{1}=a$ and $x_{2}=b$ to conclude that $T_{0}(x) \rightarrow \infty$ as $x=a$ or $b$.

\section{Application to stationary level-set equations}

In this section, we turn to investigate the stationary level-set equation (E2). We attempt to obtain a game-theoretic interpretation for this equation when $\partial \Omega$ is represented by the graph of a periodic smooth function with multiple concave parts. Our games are played in an unbounded domain.

Let the step size of space be $\epsilon>0$. Suppose that the game starts from $z \in \mathbb{R}^{2}$ and there are two players, Player I and Player II. The following set of game rules is repeated during the game.

Game rules: In the $i$-th round $(i=1,2, \ldots, N)$ with position $\eta \in \mathbb{R}^{2}$,

(1) Player I first chooses a vector $v_{i} \in \mathbb{R}^{2}$ with $\left|v_{i}\right| \leq 1$.

(2) Player II has a right to support or reverse Player I's choice; that is, Player II may take $b_{i}= \pm 1$.

(3) Once the decisions above have been made, we update the position, letting it be $\eta+\sqrt{2} \epsilon b_{i} v_{i}$.

There are two ways to proceed and end this game:

1. (Finite horizon problem:) One way is to set a fixed ending time $t \geq 0$. To be more precise, we take $N=\left[t / \epsilon^{2}\right]$. Let $y(t ; z, b, v)$ be the game state after $N$ rounds. We are then able to get $U_{0}(y(t ; z, b, v))$, which in fact depends on the initial position $z$, duration $t$, step size $\epsilon$ and certainly the decisions $b, v$ of both players. Suppose Player I wants to maximize $U_{0}(y(t ; z, b, v))$ and Player II wants to minimize it. The value function is thus defined as

$$
U^{\epsilon}(z, t)=\max _{v_{1}} \min _{b_{1}} \max _{v_{2}} \min _{b_{2}} \cdots \max _{v_{N}} \min _{b_{N}} U_{0}(y(t ; z, b, v))
$$

2. (Time optimal problem:) The other way is to consider the first exit time from $\bar{\Omega}$; namely, the game will end only when the position first leaves $\bar{\Omega}$. The ending round $N$ itself depends on the strategies of both players. We let $\tau^{\epsilon}(z ; b, v):=N \epsilon^{2}$ denote the first time of exit from $\bar{\Omega}$. If Player I attempts to minimize $\tau^{\epsilon}$ and Player II tries to maximize $\tau^{\epsilon}$, then we may define value function for this game as 


$$
T^{\epsilon}(z)=\min _{v_{1}} \max _{b_{1}} \min _{v_{2}} \max _{b_{2}} \cdots \tau^{\epsilon}(z ; b, v) .
$$

It is clear that $T^{\epsilon}(z)=0$ if $z \notin \bar{\Omega}$. We adopt the convention that $T^{\epsilon}(z)=\infty$ if the game starting from $x$ cannot be ended.

The first type of game provides us with the approximation and, as a byproduct, the existence of solutions of (E1). Notice that the uniqueness follows from the comparison principle; see [12].

Theorem 4.1. [16] Let $U^{\epsilon}$ be the value function defined in (4.1). If $U_{0}$ is bounded and uniformly continuous, then $U^{\epsilon} \rightarrow U$ locally uniformly as $\epsilon \rightarrow 0$, where $U$ is the viscosity solution of (E1).

The link between the level set and graph formulations can be easily seen from the following.

Proposition 4.2. Suppose that $u_{0}: \mathbb{R} \rightarrow \mathbb{R}$ is a uniformly continuous function and $u$ is the unique solution of (1.3). Let $U(x, y, t)=u(x, t)-y$ for all $x, y \in \mathbb{R}$ and $t \geq 0$. Then $U$ is the unique solution of (E1) satisfying $U_{0}(x, y)=u_{0}(x)-$ $y$.

Proof. The proof is straightforward. Note that the initial condition is clearly satisfied. We now show that $U$ is a subsolution of (eq: E1a). Suppose there are $\left(x_{0}, y_{0}\right) \in \mathbb{R}^{2}, t_{0}>0$ and $\phi \in C^{\infty}\left(\mathbb{R}^{2} \times(0, \infty)\right)$ such that

$$
\max _{\mathbb{R}^{2} \times(0, \infty)}(U-\phi)=(U-\phi)\left(x_{0}, y_{0}, t_{0}\right) .
$$

Since $U(x, y, t)=u(x, t)-y, y \mapsto u\left(x_{0}, t_{0}\right)-y-\phi\left(x_{0}, y, t_{0}\right)$ attains a maximum at $y_{0}$. Then it is obvious that

$$
\phi_{y}\left(x_{0}, y_{0}, t_{0}\right)=-1 .
$$

On the other hand, $(x, t) \mapsto u(x, t)-y_{0}-\phi\left(x, y_{0}, t\right)$ attains a maximum at $\left(x_{0}, t_{0}\right)$. Then by the definition of subsolutions of (1.3), we have

$$
\phi_{t}\left(x_{0}, t_{0}\right)-\frac{\left(\phi_{x x}\left(x_{0}, t_{0}\right)\right)_{+}}{1+\phi_{x}^{2}\left(x_{0}, t_{0}\right)} \leq 0,
$$

which, together with (4.3), yields

$$
\phi_{t}-|\nabla \phi| \operatorname{div}\left(\frac{\nabla \phi}{|\nabla \phi|}\right)_{+} \leq 0 \quad \text { at }\left(x_{0}, y_{0}, t_{0}\right) \text {. }
$$

The proof for supersolution verification is similar.

We now turn our attention to the game with exit time. The dynamic programming principle for this game is

$$
T^{\epsilon}(z)=\min _{|v| \leq 1} \max _{b= \pm 1} T^{\epsilon}(z+\sqrt{2} \epsilon b v)+\epsilon^{2} .
$$

for all $z$ such that for any $|v| \leq 1$, there exists $b= \pm 1$ satisfying $z+\sqrt{2} \epsilon b v \in \bar{\Omega}$. We let $T^{\epsilon}(z)=\epsilon^{2}$ if there is $\left|v_{0}\right| \leq 1$ such that $z+\sqrt{2} \epsilon b v \notin \bar{\Omega}$ for both $b= \pm 1$.

The value function $T^{\epsilon}$ is supposed to converge, as $\epsilon \rightarrow 0$, to a solution of the corresponding stationary problem like (E2) in an analogous manner. It however turns out to be quite difficult in general. As was described above, the 
main difficulty is the loss of comparison theorems due to the lack of (strict) boundary data. We aim to overcome this difficulty and present a game-based convergence theorem by using our results in the previous section on the waiting time.

To this end, we assume

(A4) the boundary $\partial \Omega:=\left\{(x, y) \in \mathbb{R}^{2}: y=u_{0}(x)\right\}$ and $\Omega=\left\{(x, y) \in \mathbb{R}^{2}\right.$ : $\left.y>u_{0}(x)\right\}$, where $u_{0}: \mathbb{R} \rightarrow \mathbb{R}$ is a periodic function fulfilling (A2)-(A3).

Let us define the relaxed limits of $T^{\epsilon}$ for any $z \in \bar{\Omega}$

$$
\bar{T}(z)=\limsup _{\epsilon \rightarrow 0} T^{\epsilon}(z) \text { and } \quad \underline{T}(z)=\liminf _{\epsilon \rightarrow 0} T^{\epsilon}(z)
$$

A natural question arising from this definition is whether $T^{\epsilon}(z)$ (also $\bar{T}$ and $\underline{T}$ ) is finite for all $z \in \Omega$. The answer is negative because of the unboundedness of the domain. Indeed, let $\Gamma=\left\{(x, y) \in \mathbb{R}^{2}: y=M\left(=\max _{I} u_{0}\right)\right\}$. Then $\Gamma$ divides the domain $\Omega$ into a half plane and countably many congruent parts. Taking one of the congruent parts, we denote

$$
\Omega_{e}=\left\{(x, y) \in I \times \mathbb{R}: u_{0}(x)<y<M\right\},
$$

which is connected due to (A4). Roughly speaking, $T^{\epsilon}(z)$ is finite when $z \in \Omega_{e}$ but is infinite when $z \notin \Omega_{e}$. In order to prove this, we need the connection between time-dependent games and the associated stationary games.

Lemma 4.3. (Game connections) Assume (A4). Let $U(z, t)$ be the unique solution of (E1) with a choice of $U_{0}$ satisfying

$$
\left\{z \in \mathbb{R}^{2}: U_{0}(z)<0\right\}=\Omega .
$$

Let $T^{\epsilon}$ be the value function defined as in (4.2). Then for any $z \in \bar{\Omega}$ and $t \geq 0$,

(1) $U(z, t)>0$ implies $t \geq \bar{T}(z)$;

(2) $U(z, t)<0$ implies $t \leq \underline{T}(z)$.

Proof. Fix $z_{0} \in \bar{\Omega}$ and $t \geq 0$ arbitrarily.

(1) Our goal is to show $\bar{T}\left(z_{0}\right) \leq t$ under the assumption that $U\left(z_{0}, t\right)>0$. We may take $\delta>0$ such that $U^{\epsilon}(z, t)>\delta$ for all $z \in B_{\delta}\left(z_{0}\right)$ owing to Theorem 4.1. It means that for any $z \in B_{\delta}\left(z_{0}\right)$ there exists a strategy $v^{z}$ of Player I such that $U_{0}\left(y\left(t ; z, v^{z}, b\right)\right)>\delta$, i.e., $y\left(t ; z, v^{z}, b\right) \notin \bar{\Omega}$ for any $b$. Note that here we abuse the notation, using $v$ and $b$ to denote strategies, which represent the sequences of choices of both players. As a result, by definition we have $T^{\epsilon}(z)<t$ for all $z \in B_{\delta}\left(z_{0}\right)$, which implies that $\bar{T}\left(z_{0}\right) \leq t$.

(2) We now prove $\underline{T}\left(z_{0}\right) \geq t$ provided that $U\left(z_{0}, t\right)<0$. Applying Theorem 4.1 as above, we have $\delta>0$ such that $U^{\epsilon}(z, t)<-\delta$ for all $z \in B_{\delta}\left(z_{0}\right)$. We thus can ensure the existence of a strategy $b^{z}$ of Player II satisfying $U_{0}\left(y\left(t ; z, v, b^{z}\right)\right)<-\delta$ for any $v$; in other words, $y\left(t ; z, v, b^{z}\right) \in \Omega$. We claim that $y\left(s ; z, v, b^{z}\right) \in \bar{\Omega}$ for all $s \leq t$. If it is not true, i.e., there exists $s_{0} \leq t$ such that $y\left(s_{0} ; z, v, b^{z}\right) \notin \bar{\Omega}$. Then Player I may keep choosing the trivial option $v=0$ for the rest of the finite horizontal game. This strategy of Player I yields $y\left(t ; z, v, b^{z}\right) \notin \bar{\Omega}$, which is clearly a contradiction. Hence, 
under the strategy $b^{z}$, we must have the first exit time $\tau_{z}>t$. It follows that $T^{\epsilon}(z)>t$ for all $z \in B_{\delta}\left(z_{0}\right)$, which in turn implies that $\underline{T}\left(z_{0}\right) \geq t$.

Lemma 4.4. (Finiteness of exit time) Assume (A4). Let $\Omega_{0}$ and $\Omega_{e}$ be defined as in (2.1) and in (4.5) respectively. Let $T^{\epsilon}$ be the value function defined as in (4.2). Then

(i) $\bar{T}(z)<\infty$ for all $z \in \Omega_{e}$;

(ii) $\bar{T}(z)=\infty$ for all $z \in \Omega_{0} \backslash \Omega_{e}$;

(iii) $\underline{T}(z) \rightarrow \infty$ if $\operatorname{dist}(z, \Gamma) \rightarrow 0$ with $z \in \Omega_{e}$.

Proof. (i) For any fixed $z=(x, y) \in \Omega_{e}$, in virtue of Proposition 2.3 , there is $t_{0}>0$ such that $u(x, t)-y>0$ for all $t>t_{0}$, which implies that $\bar{T}(z) \leq t_{0}$ by Lemma 4.3.

(ii) In terms of Proposition 2.3 and Lemma 2.2, we have $u(x, t)-M \leq 0$ for all $x \in \mathbb{R}$ and $t \geq 0$, which amounts to saying that $U(z, t)<0$ for all $z \in \Omega_{0} \backslash \Omega_{e}$. The conclusion follows again from the application of Lemma 4.3.

(iii) For any $t>0$, we may employ Theorem 3.8 to get $u(x, t)<M$ for all $x \in I$. We are now able to arbitrarily pick $y \in(u(x, t), M)$ for any $x \in I$ such that $U(x, y, t)=u(x, t)-y<0$. In view of Lemma 4.3, we are led to $\underline{T}(z) \geq t$ for $z=(x, y) \in \Omega_{e}$. This concludes the proof.

The set $\Omega_{e}$ turns out to be an effective domain for our games. The dynamic programming principle reads

$$
T^{\epsilon}(z)=\min _{|v|=1} \max _{b= \pm 1} T^{\epsilon}(z+\sqrt{2} \epsilon b v)+\epsilon^{2}
$$

for any $z \in \mathbb{R}^{2}$ provided that $T^{\epsilon}(z) \neq 0$.

One may naturally guess that the limit of $T^{\epsilon}$ is a solution of the singular boundary problem (E4). It is true but (1.10) should be interpreted in the viscosity sense, since it may not be realized in the strict sense. Indeed, for any $z_{0}=\left(x_{0}, y_{0}\right)$ on the concave piece of $\partial \Omega$, Lemma 3.1(ii) indicates that there exists $\tau_{0}>0$ such that $u\left(x_{0}, t\right)-y_{0}=u_{0}\left(x_{0}\right)-y_{0}=0$ for all $t \in\left[0, \tau_{0}\right]$. We thus have

$$
U\left(x_{0}, y, \tau_{0}\right)=U_{0}\left(x_{0}, y\right)=u_{0}\left(x_{0}\right)-y<0 \quad \text { for all } y>u_{0}\left(x_{0}\right) .
$$

By Lemma 4.3, we have $\bar{T}\left(x_{0}, y\right) \geq \tau_{0}$ and therefore $\bar{T}\left(z_{0}\right) \geq \tau_{0}$.

For this reason, in what follows we choose to characterize the limit of $T^{\epsilon}$ as a unique solution $T$ of (E3), where (1.8) is in the strict sense. For clarity and later use, let us provide the definition of viscosity solutions for a class of elliptic equations in the form:

$$
-|\nabla U| \operatorname{div}\left(\frac{\nabla U}{|\nabla U|}\right)+f(U)=0 \quad \text { in } \mathcal{O},
$$

where $\mathcal{O}$ is an open subset of $\mathbb{R}^{2}$ and $f: \mathbb{R} \rightarrow \mathbb{R}$ is a continuous nondecreasing function. It is clear that (1.6) is a special case of (4.7). 
Definition 4.1. An upper semicontinuous (resp., lower semicontinuous) function $U$ on $\mathcal{O}$ is a viscosity subsolution (resp., viscosity supersolution) of (4.7) if whenever there are $\hat{z} \in \mathcal{O}$ and a function $\varphi \in C^{2}(\mathcal{O})$ such that

$$
\begin{aligned}
& \max _{\mathcal{O}}(U-\varphi)=(U-\varphi)(\hat{z}) \\
& \quad\left(\text { resp., } \min _{\mathcal{O}}(U-\varphi)=(U-\varphi)(\hat{z})\right),
\end{aligned}
$$

the following holds:

(i) If $\nabla \varphi(\hat{z}) \neq 0$, then

$$
\begin{aligned}
& -\operatorname{tr}\left(\left(I-\frac{\nabla \varphi(\hat{z}) \otimes \nabla \varphi(\hat{z})}{|\nabla \varphi(\hat{z})|^{2}}\right) \nabla^{2} \varphi(\hat{z})\right)+f(U(\hat{z})) \leq 0 \\
& \quad\left(\text { resp., }-\operatorname{tr}\left(\left(I-\frac{\nabla \varphi(\hat{z}) \otimes \nabla \varphi(\hat{z})}{|\nabla \varphi(\hat{z})|^{2}}\right) \nabla^{2} \varphi(\hat{z})\right)+f(U(\hat{z})) \geq 0\right) .
\end{aligned}
$$

(ii) If $\nabla \varphi(\hat{z})=0$, then there exists $q \in \mathbb{R}^{2}$ with $|q| \leq 1$ such that

$$
\begin{aligned}
& -\operatorname{tr}\left((I-q \otimes q) \nabla^{2} \varphi(\hat{z})\right)+f(U(\hat{z})) \leq 0 \\
& \quad\left(\text { resp., }-\operatorname{tr}\left((I-q \otimes q) \nabla^{2} \varphi(\hat{z})\right)+f(U(\hat{z})) \geq 0\right) .
\end{aligned}
$$

Definition 4.2. A function $U$ on $\mathbb{R}^{2} \times(0, T)$ is called a viscosity solution of (4.7) if it is both a viscosity subsolution and a viscosity supersolution.

Theorem 4.5. (Strong characterization) Assume (A4). Then $\bar{T}$ and $\underline{T}$ are respectively viscosity subsolutions and supersolutions of

$$
-|\nabla T| \operatorname{div}\left(\frac{\nabla T}{|\nabla T|}\right)-1=0 \quad \text { in } \Omega_{e},
$$

with $\underline{T} \leq T_{0} \leq \bar{T}$ on $\partial \Omega_{e}$ and $\underline{T}(z), \bar{T}(z) \rightarrow \infty$ as $\operatorname{dist}(z, \Gamma) \rightarrow 0$.

Proof. The derivation of (4.8) from games is standard; consult [16, Theorem 1.3]. It is also straightforward that $\bar{T}(z), \underline{T}(z) \rightarrow \infty$ as $\operatorname{dist}(z, \Gamma) \rightarrow 0$ due to Theorem 4.4(iii). We now focus our attention on showing that $\bar{T}(z) \leq T_{0}(z)$ and $\underline{T}(z) \geq T_{0}(z)$ for any $z \in \partial \Omega_{e}$. As it is trivial to show these for the case that $z \in \partial \Omega_{e} \cap \Gamma$, we only consider the situation when $z \in \partial \Omega_{e} \backslash \Gamma$.

To this end, we fix any $z_{0}=\left(x_{0}, y_{0}\right) \in \partial \Omega_{e} \backslash \Gamma$, i.e, $y_{0}=u_{0}\left(x_{0}\right)$ with $x_{0} \in I$. If $T_{0}\left(z_{0}\right)=0$, then by Theorem $3.12 u\left(x_{0}, t\right)>u_{0}\left(x_{0}\right)=y$ or in other words, $U\left(z_{0}, t\right)>0$ for any $t>0$ small, which yields that $\bar{T}\left(z_{0}\right) \leq 0$ due to Lemma 4.3. Hence $\bar{T}\left(z_{0}\right)=\underline{T}\left(z_{0}\right)=T_{0}\left(z_{0}\right)=0$.

Suppose $T_{0}\left(z_{0}\right)>0$. We take any $t_{1}, t_{2}$ such that $t_{1}<T_{0}\left(z_{0}\right)<t_{2}$. By Corollary 3.13, there exists $\delta>0$ such that we have

$$
u\left(x, t_{1}\right)=u_{0}(x) \quad \text { and } \quad u\left(x, t_{2}\right)>u_{0}(x) \quad \text { for any } x \in\left(x_{0}-\delta, x_{0}+\delta\right) .
$$

By Theorem 3.15, we have $x \in I_{-}$. For a sufficiently small $\delta>0$, Player II can control a game trajectory starting from any $z \in B_{\delta / 2}\left(z_{0}\right) \cap \bar{\Omega}$ so that it only hits $\partial B_{\delta}\left(z_{0}\right) \cap \Omega$ before it exits from $\bar{\Omega}$. Since $U\left(\cdot, t_{1}\right)<0$ on $\partial B_{\delta}\left(z_{0}\right) \cap \Omega$, which implies that $T^{\epsilon} \geq t_{1}$ on $\partial B_{\delta}\left(z_{0}\right) \cap \Omega$ as similarly shown in the proof 
of Theorem 4.3, we may connect the game trajectories and conclude that $T^{\epsilon}(z) \geq t_{1}$ for all $z \in B_{\delta / 2}\left(z_{0}\right) \cap \bar{\Omega}$ and hence

$$
\underline{T}\left(z_{0}\right) \geq t_{1} \text {. }
$$

On the other hand, we may let $\delta>0$ be smaller if necessary to get

$$
U\left(z, t_{2}\right)>0 \text { for all } z \in B_{\delta}\left(z_{0}\right) \cap \bar{\Omega} .
$$

Applying Lemma 4.3, we end up with

$$
\bar{T}(z) \leq t_{2} \quad \text { for all } z \in B_{\delta}\left(z_{0}\right) \cap \Omega,
$$

which, together with (4.9), implies

$$
\underline{T}\left(z_{0}\right) \geq T_{0}\left(z_{0}\right) \geq \bar{T}\left(z_{0}\right)
$$

by the arbitrariness of $t_{1}$ and $t_{2}$.

Before showing a comparison principle for (E3), we first apply the classical Kružkov transform to simplify our Eq. (4.8). Let $F(s):=e^{-s}$, which is a bounded decreasing smooth function. If $T$ is a subsolution (resp. supersolution) of (4.8), then $S=F \circ T$ must be a supersolution (resp. subsolution) of

$$
-|\nabla S| \operatorname{div}\left(\frac{\nabla S}{|\nabla S|}\right)+S=0 \quad \text { in } \Omega_{e} .
$$

In addition, $0 \leq S \leq 1$.

Theorem 4.6. (Comparison with strong boundary condition) Assume (A4). Let $T_{1}$ and $T_{2}$ be respectively a sub- and a supersolution of (1.6). If $T_{1} \leq T_{2}$ on $\partial \Omega$ and $T_{1}(z), T_{2}(z) \rightarrow \infty$ as $\operatorname{dist}(z, \Gamma) \rightarrow 0$, then $T_{1}(z) \leq T_{2}(z)$ for all $z \in \Omega_{e}$.

Proof. We use the Kružkov transform $F$, setting

$$
S_{1}(x)=F \circ T_{1}(x) \text { and } S_{2}(x)=F \circ T_{2}(x) .
$$

Now $S_{1}$ and $S_{2}$ are respectively a nonnegative supersolution and a nonnegative subsolution of (4.11) with $S_{1} \geq S_{2}$ on $\partial \Omega$ and $S_{1}(z), S_{2}(z) \rightarrow 0$ as $\operatorname{dist}(z, \Gamma) \rightarrow$ 0 . Using a standard comparison argument, we get $S_{1} \geq S_{2}$ in $\Omega_{e}$, which is equivalent to $T_{1} \leq T_{2}$ in $\Omega_{e}$.

Combining Theorem 4.5 and Theorem 4.6, we obtain our main result of this section.

Theorem 4.7. (Strong characterization with convergence) Assume (A4). Let $T^{\epsilon}$ be the value function defined in (4.2) and $T_{0}$ be the waiting time of motion by positive curvature defined in (3.2). Then $T^{\epsilon} \rightarrow T$ uniformly on any compact subset of $\bar{\Omega} \backslash \Gamma$ as $\epsilon \rightarrow 0$, where $T$ is the unique continuous solution of (E3).

On the other hand, we may show that $T$ is also a weak solution of (E4) in that $T$ satisfies (1.10) in the viscosity sense.

Proposition 4.8. (Weak characterization) Assume (A4). Let $T^{\epsilon}$ be the value function defined in (4.2) and $T$ be its locally uniform limit as in Theorem 4.7. Then $T$ satisfies (E4) with (1.10) in the viscosity sense. 
Proof. Since $T$ still satisfies (1.6) in $\Omega_{e}$ and (1.9) on $\Gamma$, we only verify the other boundary condition (1.10). Moreover, the fact that $T \geq 0$ allows us to only show that $T$ is a subsolution on $\partial \Omega$. For any $z_{0} \in \partial \Omega$ such that $T\left(z_{0}\right)$ is finite, if $\bar{T}\left(z_{0}\right) \neq 0$, then there is $r>0$ such that $T^{\epsilon}(z) \geq r$ in $B_{r}\left(z_{0}\right)$. Then $T^{\epsilon}$ must satisfy the dynamic programming principle (4.4) at $z_{0}$. The rest of the proof follows exactly the same as that of the interior case.

As in the case of a bounded domain, it is not known whether the weak solutions are unique but Proposition 4.8 indicates that there is a continuous one among them. We give a comparison principle below, showing that this continuous, game related solution is maximal. The proof is less standard and is similar to that for state constraint problem [26].

Theorem 4.9. (Comparison with weak boundary conditions) Assume (A4). Suppose that $T_{1}$ and $T_{2}$ are respectively an upper semicontinuous subsolution and a continuous supersolution of (E4) with (1.10) in the viscosity sense. Then $T_{1} \leq T_{2}$ in $\bar{\Omega}$.

Proof. Thanks to the Kružkov transform, it suffices to show any supersolution $S_{1}$ and any continuous subsolution $S_{2}$ of

$$
\begin{cases}S-|\nabla S| \operatorname{div}\left(\frac{\nabla S}{|\nabla S|}\right)=0 & \text { in } \Omega_{e} \\ S(z)=1 & \text { for all } z \in \partial \Omega \\ S(z)=0 & \text { for all } z \in \Gamma\end{cases}
$$

with $0 \leq S_{1}, S_{2} \leq 1$ satisfy $S_{1} \geq S_{2}$ in $\bar{\Omega}_{e}$. We note that only (4.12) is realized in the viscosity sense. Suppose by contradiction that $\max _{\bar{\Omega}_{e}}\left(S_{2}-S_{1}\right)=: \sigma>0$. We can easily deduce a contradiction when $\max _{\bar{\Omega}_{e}}\left(S_{2}-S_{1}\right)>\max _{\partial \Omega_{e}}\left(S_{2}-S_{1}\right)$ by the standard comparison argument. We therefore assume $\max _{\partial \Omega_{e}}\left(S_{2}-\right.$ $\left.S_{1}\right)=\sigma$. It implies that there exists $z_{0} \in \partial \Omega \backslash \Gamma$ such that $S_{2}\left(z_{0}\right)-S_{1}\left(z_{0}\right)=\sigma$ since $S_{2}=S_{1}=0$ on $\Gamma$. Moreover, due to the lower semicontinuity of $S_{1}$ and continuity of $S_{2}$, there exist $x_{1}, x_{2} \in I=(a, b)$ such that

$$
\left\{z:\left(S_{2}-S_{1}\right)(z) \geq \sigma\right\} \subset\left\{\left(x, u_{0}(x)\right) \in \partial \Omega: x \in\left(x_{1}, x_{2}\right)\right\} .
$$

Taking $\mu>0$ sufficiently small, we take

$$
K_{\mu}=\left\{(x, y): x \in\left[x_{1}-\mu, x_{2}+\mu\right], y \in\left[u_{0}(x), u_{0}(x)+\mu\right]\right\}
$$

such that $K_{\mu} \subset \bar{\Omega}_{e}$ and $\Gamma \cap \partial \Omega \cap K_{\mu}=\emptyset$.

Notice that there is a unit vector $k \in \mathbb{R}^{2}$ and $c>0$ such that $B_{c r}(z+r k) \in$ $\Omega_{e}$ for any $z \in K_{\mu}$ when $r>0$ is sufficiently small. (One may let $k$ be the unit vertical vector $(0,1)$ and choose $c$ properly according to the given $u_{0}$.)

Set for any small parameter $\epsilon>0$

$$
\Phi(\xi, \eta)=S_{2}(\xi)-S_{1}(\eta)-\frac{1}{4}\left|\frac{\xi-\eta}{\epsilon}-k\right|^{4}
$$


and let $\left(\xi_{\epsilon}, \eta_{\epsilon}\right)$ be a maximizer of $\Phi$ in $\bar{\Omega}_{e} \times \bar{\Omega}_{e}$. We then have $\Phi\left(\xi_{\epsilon}, \eta_{\epsilon}\right) \geq$ $\Phi\left(z_{0}+\epsilon k, z_{0}\right)$, which can be rewritten as

$$
S_{2}\left(\xi_{\epsilon}\right)-S_{1}\left(\eta_{\epsilon}\right) \geq \frac{1}{4}\left|\frac{\xi_{\epsilon}-\eta_{\epsilon}}{\epsilon}-k\right|^{4}+S_{2}\left(z_{0}+\epsilon k\right)-S_{1}\left(z_{0}\right)
$$

or

$$
\frac{1}{4}\left|\frac{\xi_{\epsilon}-\eta_{\epsilon}}{\epsilon}-k\right|^{4} \leq S_{2}\left(\xi_{\epsilon}\right)-S_{1}\left(\eta_{\epsilon}\right)-S_{2}\left(z_{0}+\epsilon k\right)+S_{1}\left(z_{0}\right) .
$$

We therefore have $\left|\xi_{\epsilon}-\eta_{\epsilon}\right| \rightarrow 0$ as $\epsilon \rightarrow 0$. By taking a subsequence, we may assume $\xi_{\epsilon}, \eta_{\epsilon} \rightarrow z_{1}$ for some $z_{1} \in \bar{\Omega}_{e}$ as $\epsilon \rightarrow 0$. Passing to the limit in (4.14) as $\epsilon \rightarrow 0$ yields

$$
S_{2}\left(z_{1}\right)-S_{1}\left(z_{1}\right) \geq \sigma .
$$

Hence $\xi_{\epsilon}, \eta_{\epsilon} \in K_{\mu}$ when $\epsilon$ is small.

In addition, sending the limit in (4.15) enables us to get

$$
\limsup _{\epsilon \rightarrow 0} \frac{1}{4}\left|\frac{\xi_{\epsilon}-\eta_{\epsilon}}{\epsilon}-k\right|^{4} \leq S_{2}\left(z_{1}\right)-S_{1}\left(z_{1}\right)-\left(S_{2}\left(z_{0}\right)-S_{1}\left(z_{0}\right)\right) \leq 0 .
$$

We are led to

$$
\frac{\xi_{\epsilon}-\eta_{\epsilon}}{\epsilon}-k \rightarrow 0 \quad \text { as } \epsilon \rightarrow 0 .
$$

In other words, we have $\left|\xi_{\eta}-\left(\eta_{\epsilon}+\epsilon k\right)\right|<c \epsilon$, which means $\xi_{\epsilon} \in \Omega_{e}$ in view of the choice of $k$ and $c$.

Also, it follows from (4.16) that the viscosity inequality for $S_{1}$ holds at $\eta_{\epsilon}$ even if $\eta_{\epsilon} \in \partial \Omega$. In order to compare the viscosity inequalities satisfied by $S_{1}$ and $S_{2}$, we calculate the derivatives of the function $\frac{1}{4}\left|\frac{\xi_{\epsilon}-\eta}{\epsilon}-k\right|^{4}$ and denote

$$
\begin{aligned}
& p_{\epsilon}=\frac{1}{\epsilon}\left|\frac{\xi_{\epsilon}-\eta_{\epsilon}}{\epsilon}-k\right|^{2}\left(\frac{\xi_{\epsilon}-\eta_{\epsilon}}{\epsilon}-k\right) ; \\
& P_{\epsilon}=\frac{1}{\epsilon^{2}}\left|\frac{\xi_{\epsilon}-\eta_{\epsilon}}{\epsilon}-k\right|^{2} I+\frac{2}{\epsilon^{2}}\left(\frac{\xi_{\epsilon}-\eta_{\epsilon}}{\epsilon}-k\right) \otimes\left(\frac{\xi_{\epsilon}-\eta_{\epsilon}}{\epsilon}-k\right) .
\end{aligned}
$$

By the standard Crandall-Ishii lemma, there exist $X_{\epsilon}, Y_{\epsilon} \in \mathbb{R}^{2 \times 2}$ symmetric satisfying

$$
\left(p_{\epsilon}, X_{\epsilon}\right) \in \bar{J}_{\Omega_{e}}^{2,+} S_{2}\left(\xi_{\epsilon}\right) \quad \text { and } \quad\left(p_{\epsilon}, Y_{\epsilon}\right) \in \bar{J}_{\bar{\Omega}_{e}}^{2,-} S_{1}\left(\eta_{\epsilon}\right)
$$

and

$$
\left(\begin{array}{ll}
X_{\epsilon} & 0 \\
0 & -Y_{\epsilon}
\end{array}\right) \leq\left(\begin{array}{ll}
P_{\epsilon} & -P_{\epsilon} \\
-P_{\epsilon} & P_{\epsilon}
\end{array}\right)+2 \epsilon\left(\begin{array}{ll}
P_{\epsilon}^{2} & -P_{\epsilon}^{2} \\
-P_{\epsilon}^{2} & P_{\epsilon}^{2}
\end{array}\right)
$$

(We refer to [6] and [12] for the definition of semijets and more details on this lemma.) It follows immediately from the relation (4.18) that $X_{\epsilon} \leq Y_{\epsilon}$ and $X_{\epsilon} \leq P_{\epsilon}$.

Now we need to plug the calculations above in the definition of sub- and supersolutions of (4.11). We discuss two cases. 
Case $A: p_{\epsilon}=0$. We easily see that $P_{\epsilon}=0$ and therefore $X_{\epsilon} \leq 0$. Then Definition 4.1 implies that there exists $q \in \mathbb{R}^{2}$ with $|q| \leq 1$ such that

$$
S_{2}\left(\xi_{\epsilon}\right) \leq \operatorname{tr}(q \otimes q) X_{\epsilon} \leq 0,
$$

which is clearly a contradiction to (4.14).

Case $B: p_{\epsilon} \neq 0$. In this case, we apply Definition 4.1 for both $S_{1}$ and $S_{2}$ to get

$$
\begin{aligned}
& S_{2}\left(\xi_{\epsilon}\right)-\operatorname{tr}\left(\left(I-\frac{p_{\epsilon} \otimes p_{\epsilon}}{\left|p_{\epsilon}\right|^{2}}\right) X_{\epsilon}\right) \leq 0 ; \\
& S_{1}\left(\eta_{\epsilon}\right)-\operatorname{tr}\left(\left(I-\frac{p_{\epsilon} \otimes p_{\epsilon}}{\left|p_{\epsilon}\right|^{2}}\right) Y_{\epsilon}\right) \geq 0 .
\end{aligned}
$$

Taking their difference and using the fact that $X_{\epsilon} \leq Y_{\epsilon}$ give

$$
S_{2}\left(\xi_{\epsilon}\right)-S_{1}\left(\eta_{\epsilon}\right) \leq 0 .
$$

This again contradicts (4.14).

Normally, one would expect that a symmetric result also holds. In other words, it seems to be true that any continuous subsolution $T_{1}$ and lower semicontinuous supersolution $T_{2}$ of (E4) satisfy $T_{1} \leq T_{2}$ in $\bar{\Omega}_{e}$, which would imply that the weak solutions of (E4) are unique, continuous and equal to the solution $T$ obtained in Theorem 4.7. However, it is not necessarily true in general. Indeed, by taking a point $z_{0}$ on $\partial \Omega$ such that $T\left(z_{0}\right)>0$, one may then define a nonnegative function $\tilde{T}$ such that $\tilde{T}\left(z_{0}\right)<T\left(z_{0}\right)$ but $\tilde{T}(z)=T(z)$ for all $z \in \bar{\Omega}_{e} \backslash\left\{z_{0}\right\}$. It is not difficult to see that $\tilde{T}$ is a lower semicontinuous supersolution of (E4) but $T\left(z_{0}\right)>\tilde{T}\left(z_{0}\right)$. This example shows that the boundary condition (1.10) for a supersolution is too weak.

\section{Acknowledgments}

The author is grateful to Professors Y. Giga and R. V. Kohn for discussions and to Professor H. Mitake for pointing out a mistake in the first draft of the paper. The author also thanks the anonymous referee for careful reading and valuable suggestions.

\section{References}

[1] Bardi, M.: A boundary value problem for the minimum-time function. SIAM J. Control Optim. 27, 776-785 (1989)

[2] Bardi, M., Capuzzo-Dolcetta, I.: Optimal Control and Viscosity Solutions of Hamilton-Jacobi-Bellman Equations. Systems and Control: Foundations and Applications. Birkhäuser Boston, Boston (1997)

[3] Bardi, M., Soravia, P.: Hamilton-Jacobi equations with singular boundary conditions on a free boundary and applications to differential games. Trans. Am. Math. Soc. 325, 205-229 (1991) 
[4] Chen, Y.-G., Giga, Y., Goto, S.: Uniqueness and existence of viscosity solutions of generalized mean curvature flow equations. J. Differ. Geom. 33, 749-786 (1991)

[5] Chopp, D., Evans, L.C., Ishii, H.: Waiting time effects for Gauss curvature flows. Indiana Univ. Math. J. 48, 311-344 (1999)

[6] Crandall, M., Ishii, H., Lions, P.L.: User's guide to viscosity solutions of second order partial differential equations. Bull. Am. Math. Soc. 27, 1-67 (1992)

[7] Evans, L.C., James, M.R.: The Hamiltonian-Jacobi-Bellman equation for timeoptimal control. SIAM J. Control Optim. 6, 1477-1489 (1989)

[8] Evans, L.C., Spruck, J.: Motion of level sets by mean curvature. I. J. Differ. Geom. 33, 635-681 (1991)

[9] Ferrari, F., Liu, Q., Manfredi, J.J.: On the horizontal mean curvature flow for axisymmetric surfaces in the Heisenberg group. Commun. Contemp. Math. (to appear)

[10] Ferrari, F., Liu, Q., Manfredi, J.J.: On the characterization of p-harmonic functions on the Heisenberg group by mean value properties. Discrete Contin. Dyn. Syst. 34, 2779-2793 (2014)

[11] Fleming, W.H., Soner, M.H.: Controlled Markov Processes and Viscosity Solutions. Applications of Mathematics (New York), vol. 25. Springer, New York (1993)

[12] Giga, Y.: Surface Evolution Equations: A Level Set Approach. Monographs in Mathematics, vol. 99. Birkhäuser Verlag, Basel (2006)

[13] Giga, Y., Goto, S., Ishii, H., Sato, M.-H.: Comparison principle and convexity preserving properties for singular degenerate parabolic equations on unbounded domains. Indiana Univ. Math. J. 40, 443-470 (1991)

[14] Giga, Y., Liu, Q., Mitake, H.: Large-time asymptotics for one-dimensional Dirichlet problems for Hamilton-Jacobi equations with noncoercive Hamiltonians. J. Differ. Equ. 252, 1263-1282 (2012)

[15] Giga, Y., Liu, Q., Mitake, H.: Singular Neumann problems and large-time behavior of solutions of noncoercive Hamilton-Jacobi equations. Trans. Am. Math. Soc. (to appear)

[16] Kohn, R.V., Serfaty, S.: A deterministic-control-based approach to motion by curvature. Commun. Pure Appl. Math. 59, 344-407 (2006)

[17] Kohn, R.V., Serfaty, S.: A deterministic-control-based approach to fully nonlinear parabolic and elliptic equations. Commun. Pure Appl. Math. 63, 1298$1350(2010)$

[18] Korevaar, N.J.: Convex solutions to nonlinear elliptic and parabolic boundary value problems. Indiana Univ. Math. J. 32, 603-614 (1983)

[19] Liu, Q.: Fattening and comparison principle for level-set equations of mean curvature type. SIAM J. Control Optim. 49, 2518-2541 (2011) 
[20] Lasry, J.M., Lions, P.L.: Nonlinear elliptic equations with singular boundary conditions and stochastic control with state constraints. Math. Ann. 283, 583-630 (1989)

[21] Malladi, R., Sethian, J.A.: Image processing: flows under man/max curvature and mean curvature. Graph. Model Image Process. 58, 127-141 (1996)

[22] Manfredi, J.J., Parviainen, M., Rossi, J.D.: An asymptotic mean value characterization for p-harmonic functions. Proc. Am. Math. Soc. 138, 881-889 (2010)

[23] Manfredi, J.J., Parviainen, M., Rossi, J.D.: An asymptotic mean value characterization for a class of nonlinear parabolic equations related to tug-of-war games. SIAM J. Math. Anal. 42, 2058-2081 (2010)

[24] Peres, Y., Schramm, O., Sheffield, S., Wilson, D.: Tug-of-war and the infinity Laplacian. J. Am. Math. Soc. 22, 167-210 (2009)

[25] Peres, Y., Sheffield, S.: Tug of war with noise: a game theoretic view of the p-Laplacian. Duke Math. J. 145, 91-120 (2008)

[26] Soner, H.M.: Optimal control with state-space constraint. I. SIAM J. Control Optim. 24, 552-561 (1986)

Qing Liu

Department of Mathematics

University of Pittsburgh

Pittsburgh

PA 15260

USA

e-mail: qingliu@pitt.edu

Received: 16 May 2013.

Accepted: 10 December 2013. 